Luis Fernando Hernández-Moguel and Rafael Herrera*

\title{
Generalized almost even-Clifford manifolds and their twistor spaces
}

https://doi.org/10.1515/coma-2020-0108

Received November 28, 2020; accepted January 19, 2021

Abstract: Motivated by the recent interest in even-Clifford structures and in generalized complex and quaternionic geometries, we introduce the notion of generalized almost even-Clifford structure. We generalize the Arizmendi-Hadfield twistor space construction on even-Clifford manifolds to this setting and show that such a twistor space admits a generalized complex structure under certain conditions.

Keywords: generalized complex structure, even-Clifford structure, twistor space

MSC: 53D18, 53C28, 53C15, 15A66, 32L25

\section{Introduction}

Generalized complex, quaternionic and exceptional geometries have attracted attention in mathematics and physics for the last two decades $[1,7,8,14,16,20,24,26,27,29,30,34,39,43]$. Pantilie [34] introduced the notion of generalized quaternionic manifold as well as the corresponding twistor space endowed with a connection-dependent generalized almost complex structure, whose integrability was discussed by Deschamps [17]. Recently, there has been some interest in Riemannian manifolds admitting even-Clifford structures $[2-5,11-13,15,18,22,23,25,28,31-33,35-38,41]$ and, in particular, Arizmendi and Hadfield [5] constructed a twistor space for even-Clifford manifolds. Motivated by these results, we define generalized evenClifford manifolds, which include generalized complex and quaternionic manifolds, and consider the construction of a twistor space endowed with a generalized complex structure by means of a suitable connection on the relevant principal bundle.

The paper is organized as follows. In Section 2, we recall standard material about generalized complex manifolds and Clifford algebras. In Section 3, we introduce the notion of generalized even-Clifford structure. In Section 4, we present the construction of the twistor space and its generalized almost complex structure. In Section 5 , we prove the main theorem regarding the integrability of the generalized almost complex structure under certain conditions.

\section{Preliminaries}

In this section we recall some concepts of generalized complex geometry [20] and Clifford algebras [19].

Luis Fernando Hernández-Moguel: UNAM and Centro de Investigación en Matemáticas, A. P. 402, Guanajuato, Gto., C.P. 36000, México, E-mail: hmoguel@cimat.mx

*Corresponding Author: Rafael Herrera: Centro de Investigación en Matemáticas, A. P. 402, Guanajuato, Gto., C.P. 36000, México, E-mail: rherrera@cimat.mx 


\subsection{Generalized (almost) complex structures.}

Let $M$ be a $2 n$-dimensional smooth manifold. The generalized tangent bundle of $M$ is

$$
\mathbb{T} M=T M \oplus T^{\star} M,
$$

together with the natural pseudo-Riemannian metric of signature $(2 n, 2 n)$ given by the canonical pairing between vectors and 1-forms

$$
\langle X+\alpha, Y+\beta\rangle=\frac{\alpha(Y)+\beta(X)}{2},
$$

for $X, Y \in \Gamma(T M)$ and $\alpha, \beta \in \Gamma\left(T^{\star} M\right)$. Given a generalized tangent vector $X=X+\alpha$, we will use the subscripts $v$ and ${ }_{f}$ to denote the vector and form parts, i.e.

$$
\begin{aligned}
& X_{v}:=\quad X \in T M, \\
& X_{f}:=\quad \alpha \in T^{\star} M .
\end{aligned}
$$

A generalized almost complex structure on $M$ is an endomorphism

$$
\mathcal{J}: \mathbb{T} M \longrightarrow \mathbb{T} M
$$

such that

$$
\jmath^{2}=-\operatorname{Id}_{\mathbb{T} M}
$$

and is orthogonal with respect to $\langle\cdot, \cdot\rangle$.

In order to define the integrability of such structures, a new Nijenhuis tensor was defined using the Courant bracket [20]. The Courant bracket is defined as

$$
\llbracket X+\alpha, Y+\beta \rrbracket=[X, Y]+\mathcal{L}_{X} \beta-\mathcal{L}_{Y} \alpha-\frac{1}{2} d\left(i_{X} \beta-i_{Y} \alpha\right),
$$

where $[X, Y]$ is the Lie bracket and $\mathcal{L}$ denotes the Lie derivative.

The generalized Nijenhuis tensor is defined as follows

$$
\mathcal{N}^{\mathcal{J}}(x, y)=\llbracket \partial x, \partial y \rrbracket-\partial \llbracket \partial x, y \rrbracket-\partial \llbracket x, \partial y \rrbracket-\llbracket x, y \rrbracket,
$$

where $x, y \in \Gamma(\mathbb{T} M)$.

Definition 2.1. A generalized almost complex structure $\mathcal{J}$ on a smooth manifold is integrable if $\mathcal{N}^{\mathfrak{d}}=0$.

Examples. [20]

1. Let $J: T M \rightarrow T M$ be a complex structure on $M$. The bundle endomorphism $\mathcal{J}_{J}: T M \oplus T^{\star} M \rightarrow T M \oplus T^{\star} M$ defined by

$$
J_{J}(X+\alpha)=J X-J^{\star} \alpha
$$

i.e.

$$
J_{J}=\left(\begin{array}{cc}
J & 0 \\
0 & -J^{\star}
\end{array}\right),
$$

where $X+\alpha \in \Gamma(\mathbb{T} M)$ and $J^{\star}$ denotes the induced transformation on forms, is a generalized complex structure, i.e. $\mathcal{N}^{\mathcal{J}_{J}}=0$.

2. Let $\omega$ be a symplectic structure on $M$ considered as a linear isomorphism $\omega: V \rightarrow V^{\star}$ such that its dual map satisfies $\omega^{\star}=-\omega$. The bundle endomorphism $\mathcal{J}_{\omega}: T M \oplus T^{*} M \rightarrow T M \oplus T^{\star} M$ defined by

$$
\mathcal{J}_{\omega}(X+\alpha)=\omega(X)-\omega^{-1}(\alpha),
$$

i.e.

$$
\partial_{\omega}=\left(\begin{array}{cc}
0 & -\omega^{-1} \\
\omega & 0
\end{array}\right),
$$

where $X+\alpha \in \Gamma(\mathbb{T} M)$, is a generalized complex structure, i.e. $\mathcal{N}^{\mathcal{J}_{\omega}}=0$. 
In [21], Gualtieri introduced the generalized torsion for certain differential operators which he called generalized connections. Motivated by such an object, Deschamps [17] considered the following generalized torsion tensor

$$
\mathcal{T}(x, y, z):=\left\langle\nabla_{x} y-\nabla_{y} x-\llbracket x, y \rrbracket, z\right\rangle+\frac{1}{2}\left(\left\langle\nabla_{z} x, y\right\rangle-\left\langle\nabla_{z} y, x\right\rangle\right),
$$

where $x, y, z \in \mathbb{T} M$,

$$
\nabla_{x} y:=\nabla_{x_{v}} y
$$

and $\nabla$ is the covariant derivative on $\mathbb{T} M$ induced by a connection on the principal bundle of orthonormal frames of $\mathbb{T} M$ with respect to the canonical pairing $\langle\cdot, \cdot\rangle$.

Remark. If $\nabla$ is a torsion free covariant derivative on the tangent bundle $T M$ of a manifold $M$, then the generalized torsion $\mathcal{T}$ of the induced connection on $T M \oplus T^{\star} M$ also vanishes identically.

Just as in the classical case, one can prove the following.

Lemma 2.1. Let $M$ be a differentiable manifold and $\nabla$ the covariant derivative of a connection on $\mathbb{T} M$. Let $\bar{S}$ be a local section of $\operatorname{End}(\mathbb{T} M)$ around $x \in M$ such that $\bar{S}^{2}=-I d_{\mathbb{T} M}$, it is orthogonal with respect to the canonical pairing $\langle\cdot, \cdot\rangle$ and $(\nabla \bar{S})_{x}=0$. Then the Nijenhuis tensor $\mathcal{N}^{\bar{S}}$ of $\bar{S}$ at the point $x$ satisfies

$$
\left\langle\mathcal{N}^{\bar{S}}(x, y), z\right\rangle=\mathcal{T}(x, y, z)-\mathcal{T}(x, \bar{S} y, \bar{S} z)-\mathcal{T}(\bar{S} x, y, \bar{s} z)-\mathcal{T}(\bar{S} x, \bar{S} y, z) .
$$

Proof. Let $X, y, z$ differentiable sections of $\mathbb{T} M, x \in M$ and $(\nabla \bar{S})_{x}=0$. At the point $x$, we have

$$
\begin{aligned}
-\mathcal{T}(X, \bar{S} y, \bar{S} z)= & -\left\langle\nabla_{x} \bar{S} y-\nabla_{\bar{S} y} x-\llbracket x, \bar{S} y \rrbracket, \bar{S} z\right\rangle-\frac{1}{2}\left(\left\langle\nabla_{\bar{S} z} x, \bar{S} y\right\rangle-\left\langle\nabla_{\bar{S} z} \bar{S} y, x\right\rangle\right) \\
= & -\left\langle\left(\nabla_{x} \bar{S}\right) y+\bar{S} \nabla_{x} y-\nabla_{\bar{S} y} x-\llbracket x, \bar{S} y \rrbracket, \bar{S} z\right\rangle \\
& -\frac{1}{2}\left(\left\langle\nabla_{\bar{S} z} x, \bar{S} y\right\rangle-\left\langle\left(\nabla_{\bar{S} z} \bar{S}\right) y+\bar{S} \nabla_{\bar{S} z} Y, x\right\rangle\right) \\
= & -\left\langle\bar{S} \nabla_{x} y-\nabla_{\bar{S} y} x-\llbracket x, \bar{S} y \rrbracket, \bar{S} z\right\rangle-\frac{1}{2}\left(\left\langle\nabla_{\bar{S} z} x, \bar{S} y\right\rangle-\left\langle\bar{S} \nabla_{\bar{S} z} Y, x\right\rangle\right) \\
= & -\left\langle\bar{S}\left(\nabla_{x} y+\bar{S} \nabla_{\bar{S} y} x+\bar{S} \llbracket x, \bar{S} y \rrbracket\right), \bar{S} z\right\rangle-\frac{1}{2}\left(\left\langle\nabla_{\bar{S} z} x, \bar{S} y\right\rangle-\left\langle\bar{S} \nabla_{\bar{S} z} Y, x\right\rangle\right) \\
= & \left\langle-\nabla_{x} y-\bar{S} \nabla_{\bar{S} y} x-\bar{S} \llbracket x, \bar{S} y \rrbracket, z\right\rangle-\frac{1}{2}\left\langle\nabla_{\bar{S} z} x, \bar{S} y\right\rangle+\frac{1}{2}\left\langle\bar{S} \nabla_{\bar{S} z} Y, x\right\rangle .
\end{aligned}
$$

Similarly,

$$
\begin{aligned}
& -\mathcal{T}(\bar{S} x, y, \bar{S} z)=\left\langle\bar{S} \nabla_{\bar{S} x} y+\nabla_{y} x-\bar{S} \llbracket \bar{S} x, y \rrbracket, z\right\rangle+\frac{1}{2}\left\langle\nabla_{\bar{S} z} x, \bar{S} y\right\rangle-\frac{1}{2}\left\langle\bar{S} \nabla_{\bar{S} z} y, x\right\rangle \\
& -\mathcal{T}(\bar{S} x, \bar{S} y, z)=\left\langle-\bar{S} \nabla_{\bar{S} x} y+\bar{S} \nabla_{\bar{S} y} x+\llbracket \bar{S} x, \bar{S} y \rrbracket, z\right\rangle-\frac{1}{2}\left\langle\nabla_{z} x, y\right\rangle+\frac{1}{2}\left\langle\nabla_{z} y, x\right\rangle .
\end{aligned}
$$

Thus,

$$
\mathcal{T}(x, y, z)-\mathcal{T}(x, \bar{S} y, \bar{S} z)-\mathcal{T}(\bar{S} x, y, \bar{S} z)-\mathcal{T}(\bar{S} x, \bar{S} y, z)=\langle\llbracket \bar{S} x, \bar{S} y \rrbracket-\bar{S} \llbracket \bar{S} x, y \rrbracket-\bar{S} \llbracket x, \bar{S} y \rrbracket-\llbracket x, y \rrbracket, z\rangle .
$$

\subsection{Clifford algebra, spin group and a real Grassmannian}

Let $C l_{n}$ denote the $2^{n}$-dimensional real Clifford algebra generated by the orthonormal vectors $e_{1}, e_{2}, \ldots, e_{n} \in$ $\mathbb{R}^{n}$ subject to the relations $e_{i} e_{j}+e_{j} e_{i}=-2 \delta_{i j}$, and $\mathbb{C} l_{n}=C l_{n} \otimes_{\mathbb{R}} \mathbb{C}$ its complexification [19]. The even Clifford subalgebra $C l_{r}^{0}$ is defined as the (+1)-eigenspace of the involution of $C l_{r}$ induced by the map $-\mathrm{Id}_{\mathbb{R}^{r}}$. Recall that $\mathrm{Cl}_{2}^{0} \cong \mathbb{C}$ and $\mathrm{Cl}_{3}^{0} \cong \mathbb{H}$. The Spin group $\operatorname{Spin}(n) \subset C l_{n}$ is the subset

$$
\operatorname{Spin}(n)=\left\{x_{1} x_{2} \cdots x_{2 l-1} x_{2 l}\left|x_{j} \in \mathbb{R}^{n},\right| x_{j} \mid=1, l \in \mathbb{N}\right\},
$$


endowed with the product of the Clifford algebra. It is a Lie group and its Lie algebra is

$$
\mathfrak{s p i n}(n)=\operatorname{span}\left\{e_{i} e_{j} \mid 1 \leq i<j \leq n\right\} .
$$

For future use, we reproduce the following lemma.

Lemma 2.2. [5] Let $A \in \mathfrak{s p i n}(r) \subset C l_{r}^{0}$. Then, $A^{2}=-1$ if and only if there exist orthonormal vectors $v_{1}, v_{2} \in \mathbb{R}^{r}$ such that $A=v_{1} \cdot v_{2}$. In other words, the set of square roots of $-1 \in C l_{r}^{0}$ is equal to the Grassmanian $\tilde{\mathbb{G}} r_{2}\left(\mathbb{R}^{r}\right)$ of oriented 2-planes in $\mathbb{R}^{r}$.

Since every point of $\tilde{\mathbb{G}} r_{2}\left(\mathbb{R}^{r}\right)$ is identified with a bivector, we can consider

$$
\tilde{\mathbb{G}} r_{2}\left(\mathbb{R}^{r}\right) \subset \mathfrak{s p i n}(r) \subset C l_{r}^{0} .
$$

\section{Generalized even-Clifford structures}

\subsection{Generalized linear even-Clifford structures}

Definition 3.1. Let $\left(e_{1}, \ldots, e_{r}\right)$ be an orthonormal basis of $\mathbb{R}^{r}$ with respect to the standard inner product, and $V$ a real vector space.

- A generalized linear even-Clifford structure of rank $r$ on $V$, or generalized almost $\mathrm{Cl}_{r}^{0}$ structure, is an algebra representation

$$
\varphi: C l_{r}^{0} \longrightarrow \operatorname{End}\left(V \oplus V^{\star}\right)
$$

such that each bivector $e_{i} e_{j}, 1 \leq i<j \leq r$, is mapped to a linear complex structure

$$
\begin{aligned}
\varphi\left(e_{i} e_{j}\right)=\mathcal{J}_{i j}: V \oplus V^{\star} & \longrightarrow V \oplus V^{\star}, \\
\partial_{i j}^{2} & =-\operatorname{Id}_{V \oplus V^{*}},
\end{aligned}
$$

which is orthogonal with respect to the canonical pairing of $V \oplus V^{\star}$,

$$
\left\langle\partial_{i j}(X+\xi), \partial_{i j}(Y+\eta)\right\rangle=\langle X+\xi, Y+\eta\rangle,
$$

where $X, Y \in V$ and $\xi, \eta \in V^{\star}$.

\section{Examples.}

1. A quaternionic structure $(I, J, K)$ on $\mathbb{R}^{4 m}$ induces a generalized linear $C l_{3}^{0}$ structure on $\mathbb{R}^{4 m} \oplus\left(\mathbb{R}^{4 m}\right)^{\star}$ as follows

$$
\left(\mathcal{J}_{I}=\begin{array}{cc}
I & 0 \\
0 & -I^{\star}
\end{array}\right), \quad \mathcal{J}_{J}=\left(\begin{array}{cc}
J & 0 \\
0 & -J^{\star}
\end{array}\right), \quad \mathcal{J}_{K}=\left(\begin{array}{cc}
K & 0 \\
0 & -K^{\star}
\end{array}\right) .
$$

2. A quaternion-Hermitian structure $(I, J, K)$ on $\mathbb{R}^{4 m}$ with respect to the standard inner product $(\cdot, \cdot)$ induces a generalized linear $C l_{3}^{0}$ structure on $\mathbb{R}^{4 m} \oplus\left(\mathbb{R}^{4 m}\right)^{\star}$ by setting $\omega_{I}=(I \cdot, \cdot) \omega_{J}=(J \cdot, \cdot), \omega_{K}=(K \cdot, \cdot)$, and

$$
\mathcal{J} \omega_{I}=\left(\begin{array}{cc}
0 & -\omega_{I}^{-1} \\
\omega_{I} & 0
\end{array}\right), \quad J \omega_{J}=\left(\begin{array}{cc}
0 & -\omega_{J}^{-1} \\
\omega_{J} & 0
\end{array}\right), \quad J \omega_{K}=\left(\begin{array}{cc}
0 & -\omega_{K}^{-1} \\
\omega_{K} & 0
\end{array}\right) .
$$

3. Let $m_{1}, m_{2}$ be positive integers. Consider a quaternion-Hermitian structure $\left(I_{1}, J_{1}, K_{1}\right)$ on $\mathbb{R}^{4 m_{1}}$ and a quaternion-Hermitian structure $\left(I_{2}, J_{2}, K_{2}\right)$ on $\mathbb{R}^{4 m_{2}}$. Consider also the corresponding symplectic forms $\omega_{I_{1}}, \omega_{J_{1}}, \omega_{K_{1}}, \omega_{I_{2}}, \omega_{J_{2}}, \omega_{J_{2}}$. We can define four generalized linear $C l_{4}^{0}$ structure on

$$
\mathbb{R}^{4 m_{1}+4 m_{2}} \oplus\left(\mathbb{R}^{4 m_{1}+4 m_{2}}\right)^{\star}=\left[\mathbb{R}^{4 m_{1}} \oplus\left(\mathbb{R}^{4 m_{1}}\right)^{\star}\right] \oplus\left[\mathbb{R}^{4 m_{2}} \oplus\left(\mathbb{R}^{4 m_{2}}\right)^{\star}\right] .
$$


Namely

$$
\begin{aligned}
& \mathcal{J}_{12}^{(1)}=\mathcal{J}_{K_{1}} \oplus\left(-\mathcal{J}_{K_{2}}\right), \quad \mathcal{J}_{13}^{(1)}=\left(-\mathcal{J}_{J_{1}}\right) \oplus \mathcal{J}_{J_{2}}, \\
& \partial_{14}^{(1)}=\mathcal{J}_{I_{1}} \oplus\left(-\mathcal{J}_{I_{2}}\right), \quad \partial_{23}^{(1)}=\mathcal{J}_{I_{1}} \oplus \mathcal{J}_{I_{2}} \text {, } \\
& J_{24}^{(1)}=\mathcal{J}_{J_{1}} \oplus \mathcal{J}_{J_{2}}, \quad J_{34}^{(1)}=\mathcal{J}_{K_{1}} \oplus \mathcal{J}_{K_{2}}, \\
& \mathcal{J}_{12}^{(2)}=\mathcal{J}_{K_{1}} \oplus\left(-\mathcal{J}_{\omega_{K_{2}}}\right), \quad \mathcal{J}_{13}^{(2)}=\left(-\mathcal{J}_{J_{1}}\right) \oplus \mathcal{J}_{\omega_{J_{2}}} \text {, } \\
& \partial_{14}^{(2)}=\mathcal{J}_{I_{1}} \oplus\left(-\mathcal{J} \omega_{I_{2}}\right), \quad \partial_{23}^{(2)}=\mathcal{J}_{I_{1}} \oplus \mathcal{\partial} \omega_{I_{2}}, \\
& J_{24}^{(2)}=\mathcal{J}_{J_{1}} \oplus \mathcal{\partial} \omega_{J_{2}}, \quad J_{34}^{(2)}=\mathcal{J}_{K_{1}} \oplus \mathcal{\partial} \omega_{K_{2}}, \\
& \mathcal{J}_{12}^{(3)}=\mathcal{J}_{\omega_{K_{1}}} \oplus\left(-\mathcal{J}_{K_{2}}\right), \quad \mathcal{J}_{13}^{(3)}=\left(-\mathcal{J}_{\omega_{J_{1}}}\right) \oplus \mathcal{J}_{J_{2}}, \\
& J_{14}^{(3)}=\mathcal{J}_{\omega_{I_{1}}} \oplus\left(-\mathcal{J}_{I_{2}}\right), \quad J_{23}^{(3)}=\mathcal{J}_{\omega_{I_{1}}} \oplus \mathcal{J}_{I_{2}}, \\
& J_{24}^{(3)}=\mathcal{J}_{\omega_{J_{1}}} \oplus \mathcal{J}_{J_{2}}, \quad \partial_{34}^{(3)}=\mathcal{J}_{\omega_{K_{1}}} \oplus \mathcal{J}_{K_{2}} \text {, } \\
& \mathcal{J}_{12}^{(4)}=\mathcal{\partial} \omega_{K_{1}} \oplus\left(-\mathcal{\partial} \omega_{K_{2}}\right), \quad \partial_{13}^{(4)}=\left(-\mathcal{\partial} \omega_{J_{1}}\right) \oplus \mathcal{J} \omega_{J_{2}}, \\
& J_{14}^{(4)}=\mathcal{J}_{\omega_{I_{1}}} \oplus\left(-\mathcal{J}_{\omega_{I_{2}}}\right), \quad J_{23}^{(4)}=\mathcal{J}_{\omega_{I_{1}}} \oplus \mathcal{J} \omega_{I_{2}}, \\
& \mathcal{J}_{24}^{(4)}=\mathcal{J} \omega_{J_{1}} \oplus \mathcal{J} \omega_{J_{2}}, \quad \mathcal{J}_{34}^{(4)}=\mathcal{J} \omega_{K_{1}} \oplus \mathcal{J} \omega_{K_{2}} \text {. }
\end{aligned}
$$

\section{Remarks.}

- The definition of generalized linear even-Clifford structure does not depend on the orthonormal basis of $\mathbb{R}^{r}$.

- Given that $C l_{r}^{0}$ is a simple algebra with unit, the Lie algebra $\mathfrak{s p i n}(r)$ is embedded by $\varphi$ into $\operatorname{End}\left(V \oplus V^{\star}\right)$.

- Let $\widehat{\operatorname{Spin}(r)}=: \varphi(\operatorname{Spin}(r)) \subset \operatorname{End}\left(V \oplus V^{\star}\right)$. The group that preserves the almost $C l_{r}^{0}$ structure is the following normalizer subgroup

$$
N_{O\left(V \oplus V^{\star}\right)}(\widehat{\operatorname{Spin}(r)})
$$

\subsection{Generalized almost even-Clifford structures on manifolds.}

Definition 3.2. Let $r \geq 2$.

- A rank $r$ generalized almost even-Clifford structure on a smooth manifold $M$ is a smoothly varying choice of a rank $r$ generalized linear even-Clifford structure on the generalized tangent space spaces of $M$.

- A smooth manifold carrying a generalized almost even-Clifford hermitian structure will be called an generalized almost even-Clifford manifold, or a generalized almost $\mathrm{Cl}_{r}^{0}$-manifold for short.

This definition can be rephrased as follows. There exists a (locally defined) Riemannian vector bundle $F \rightarrow M$ of rank $r$ such that the corresponding Clifford bundle $\mathrm{Cl}^{0}(F) \rightarrow M$ is globally defined, and an injective algebra bundle homomorphism

$$
\varphi: C l^{0}(F) \longrightarrow \operatorname{End}(\mathbb{T} M)
$$

such that for every $x \in M$

$$
\varphi_{x}: C l^{0}\left(F_{X}\right) \longrightarrow \operatorname{End}\left(\mathbb{T}_{x} M\right)
$$

is a generalized linear even-Clifford structure.

\section{Examples.}

1. A generalized almost $\mathrm{Cl}_{2}^{0}$-manifold is the same as a generalized almost complex manifold.

2. A generalized almost $\mathrm{Cl}_{3}^{0}$-manifold is the same as a generalized almost quaternionic manifold.

3. We know that almost complex manifolds and almost quaternionic manifolds provide examples supporting generalized complex and generalized quaternionic structures on their generalized tangent spaces respectively $[20,34]$. 
4. An almost even-Clifford Hermitian manifold [4, 31] is a Riemannian manifold with a (locally) defined Riemannian vector bundle $F$ and an algebra bundle map

$$
\varphi: C l^{0}(F) \longrightarrow \operatorname{End}(T M),
$$

such that with respect to a local orthonormal frame $\left(e_{1}, \ldots, e_{r}\right)$ of $F$, the endomorphisms $J_{i j}=\varphi\left(e_{i} e_{j}\right)$ are orthogonal and satisfy the Clifford relations. Thus, an almost even-Clifford Hermitian manifold admits a generalized almost even-Clifford structure given by the local endomorphisms

$$
\left(\begin{array}{cc}
J_{i j} & 0 \\
0 & -J_{i j}^{\star}
\end{array}\right) \in \operatorname{End}(\mathbb{T} M) \text {. }
$$

5. The product of two hyperkähler manifolds admits four generalized almost $\mathrm{Cl}_{4}^{0}$ structures. Namely, the four possibilities listed in Example 3 of Subsection 3.1 using the sets of generalized complex structures on each factor induced by either the threre global complex structures or the three global symplectic structures. Note that, since they are given in blocks, every single one of these structures is integrable.

Let $Q$ denote the sub-bundle of $\operatorname{End}(\mathbb{T} M)$ whose fibre at each point of $M$ is the isomorphic image of $\mathfrak{s p i n}(r)$. An almost $\mathrm{Cl}_{r}^{0}$-structure induces a reduction of structure to the subgroup

$$
G_{r}=N_{O(\operatorname{dim}(M), \operatorname{dim}(M))}(\widehat{\operatorname{Spin}(r)}) \subset O(\operatorname{dim}(M), \operatorname{dim}(M)),
$$

where $\widehat{\operatorname{Spin}(r)}$ is the image of $\operatorname{Spin}(r) \subset C l_{r}^{0}$ in $\operatorname{End}(\mathbb{T} M)$. Let $P_{G_{r}}$ be the principal bundle over $M$ with such a reduced structure group. From now on, we fix a connection on $P_{G_{r}}$ which, by definition, is compatible with the canonical pairing (viewed as a metric) and the generalized almost $\mathrm{Cl}_{r}^{0}$ structure. For instance, such a connection will induce a covariant derivative on sections of $\operatorname{End}(\mathbb{T} M)$ which will make $\mathcal{Q}$ parallel.

Note that there exists a homomorphism

$$
\sigma: G_{r}=N_{S O(\operatorname{dim}(M), \operatorname{dim}(M))}(\widehat{\operatorname{Spin}(r)}) \longrightarrow H_{r}:=\left\{\begin{array}{l}
\operatorname{Spin}(r), \\
\operatorname{SO}(r), \\
\mathbb{P} S O(r),
\end{array} \text { depending on } r \text { and } \operatorname{dim}(M),\right.
$$

so that we have a principal fibre bundle over $M$ with structure group $H_{r}$,

$$
P_{H_{r}}=P_{G_{r}} \times_{\sigma} H_{r} \longrightarrow M .
$$

\section{The twistor space construction}

The construction of the twistor space for a generalized almost even-Clifford manifold of arbitrary rank $r$ emulates the one of Arizmendi and Hadfield [5] for even-Clifford structures.

\subsection{The fibre: a real Grassmannian}

Let $r \geq 2$ and

$$
\tilde{\mathbb{G}} r_{2}\left(\mathbb{R}^{r}\right)=\frac{S O(r)}{S O(2) \times S O(r-2)}
$$

denote the real Grassmannian of oriented 2-planes in $\mathbb{R}^{r}$. This real Grassmannian has a natural complex structure since it is a Hermitian symmetric space. The complex structure $I$ on $\tilde{\mathbb{G}} r_{2}\left(\mathbb{R}^{r}\right)$ can be described using Clifford multiplication as follows. Let $S \in \tilde{\mathbb{G}} r_{2}\left(\mathbb{R}^{r}\right)$, which for a suitable frame $\left(e_{1}, \ldots, e_{r}\right)$ of $\mathbb{R}^{r}$ can be written as

$$
S=e_{1} \wedge e_{2}=e_{1} \cdot e_{2}
$$


The tangent space to $\tilde{\mathbb{G}} r_{2}\left(\mathbb{R}^{r}\right)$ at $e_{1} e_{2}$ can be identified with

$$
\begin{aligned}
T_{S} \tilde{\mathbb{G}} r_{2}\left(\mathbb{R}^{r}\right) & =\operatorname{span}\left(\left\{e_{i} e_{j} \mid i \in\{1,2\}, j \in\{3, \ldots, r\}\right\}\right) \\
& =\left\{\sum_{s=3}^{r}\left(\alpha_{s} e_{1} \cdot e_{s}+\beta_{s} e_{2} \cdot e_{s}\right) \mid \alpha_{s}, \beta_{s} \in \mathbb{R}\right\},
\end{aligned}
$$

and the almost complex structure $I_{S}$ is thus given by

$$
I_{S}(v)=S \cdot v
$$

where $v \in T_{S} \tilde{\mathbb{G}} r_{2}\left(\mathbb{R}^{r}\right)$.

\subsection{The twistor space}

Using the standard action $\rho_{r}$ of $H_{r}$ on the Hermitian symmetric space $\tilde{\mathbb{G}} r_{2}\left(\mathbb{R}^{r}\right)$, we can associate the fiber bundle

$$
\pi: Z \cong P_{H_{r}} \times \rho_{r} \tilde{\mathbb{G}} r_{2}\left(\mathbb{R}^{r}\right) \longrightarrow M
$$

which we will call the twistor space of the generalized almost $C l_{r}^{0}$-manifold $M$. In fact, for $x \in M$, the fiber $Z_{x}$ can also be described as

$$
Z_{X}=\left\{\mathcal{J}=\sum_{1 \leq i<j \leq r} a_{i j} \mathcal{J}_{i j} \in \operatorname{End}\left(\mathbb{T}_{x} M\right) \mid \mathcal{J}^{2}=-\operatorname{Id}_{\mathbb{T}_{x} M}, a_{i j} \in \mathbb{R}\right\} .
$$

which is really $\varphi\left(\tilde{\mathbb{G}} r_{2}\left(\mathbb{R}^{r}\right)\right)$, and we have $Z \subset \operatorname{End}(\mathbb{T} M)$. Under this identification, $\varphi$ becomes an inclusion map.

\subsection{The generalized tangent bundle of the twistor space}

A given connection on $P_{G_{r}}$ induces connections on all its associated bundles. This applies, in particular, to $P_{H_{r}}$ and $Z$ so that, at $S \in Z$,

$$
T_{S} Z=V_{S} \oplus H_{S},
$$

where $V_{S}=\operatorname{ker}\left(d \pi_{S}\right)$ is the tangent space to the fiber at $S \in Z$, and $H_{S}$ is the corresponding horizontal subspace at $S$ isomorphic to $T_{\pi(S)} M$ under $d \pi_{S}$. Such a decomposition implies

$$
T_{S}^{\star} Z=V_{S}^{\star} \oplus H_{S}^{\star},
$$

where

$$
\begin{aligned}
V_{S}^{\star} & =\operatorname{Ann}\left(H_{S}\right), \\
H_{S}^{\star} & =\operatorname{Ann}\left(V_{S}\right) .
\end{aligned}
$$

Thus, the generalized tangent bundle $\mathbb{T} Z=T Z \oplus T^{\star} Z$ splits as follows,

$$
\mathbb{T}_{S} Z=\left(V_{S} \oplus H_{S}\right) \oplus\left(V_{S}^{\star} \oplus H_{S}^{\star}\right),
$$

Let us define

$$
\begin{aligned}
\mathbb{V}_{S} & :=V_{S} \oplus V_{S}^{\star}, \\
\mathbb{H}_{S} & :=\quad H_{S} \oplus H_{S}^{\star} .
\end{aligned}
$$

We need to define a map

$$
\widetilde{d \pi}: T^{\star} Z \longrightarrow T^{\star} M
$$


such that

$$
V_{S}^{\star}=\operatorname{ker}\left(\widetilde{d \pi_{S}}\right)
$$

and

$$
\left.\widetilde{d \pi}_{S}\right|_{H_{S}^{\star}}: H_{S}^{\star} \longrightarrow T_{\pi(S)}^{\star} M
$$

is a linear isomorphism. Recall that for each $S \in Z_{\pi(S)}$

$$
\left.d \pi_{S}\right|_{H_{S}}: H_{S} \longrightarrow T_{\pi(S)} M
$$

is an isomorphism. It induces an isomorphism by pullback

$$
\left(\left.d \pi_{S}\right|_{H_{S}}\right)^{\star}: T_{\pi(S)}^{\star} M \longrightarrow H_{S}^{\star}
$$

so that we can define, for any $\eta \in V_{S}^{\star}$,

$$
\widetilde{d \pi}_{S}(\eta):=0
$$

and, for any $\alpha \in H_{S}^{\star}$,

$$
\widetilde{d \pi}_{S}(\alpha):=\left(\left(\left.d \pi_{S}\right|_{H_{S}}\right)^{\star}\right)^{-1}(\alpha) .
$$

Thus, we have a connection dependent generalized projection map

$$
\widehat{d \pi}_{S}:=d \pi_{S} \oplus \widetilde{d \pi}_{S}: T_{S} Z \oplus T_{S}^{\star} Z \longrightarrow T_{\pi(S)} M \oplus T_{\pi(S)}^{\star} M,
$$

which restricts to an isomorphism

$$
\left.\widehat{d \pi}_{S}\right|_{\mathbb{H}_{S}}: \mathbb{H}_{S} \longrightarrow \mathbb{T}_{\pi(S)} M
$$

whose inverse, for notational sake, will generally be denoted by $\widehat{d \pi}_{S}^{-1}$.

Definition 4.1. According to the decomposition $\mathbb{T} Z=H+H^{\star}+V+V^{\star}$ we will consider the following types of fields:

- A field $X \in \Gamma(H)$ is called a horizontal vector field.

- A horizontal vector field $X \in \Gamma(H)$ which is the horizontal lift of a vector field on $M$ is called basic.

- A field $\alpha \in \Gamma\left(H^{\star}\right)$ will be called a horizontal differential 1-form.

- A differential 1-form $\alpha$ which is the pullback under $\pi$ of a 1-form $\bar{\alpha}$ on $M$ will be called basic.

- A field $\eta \in \Gamma\left(V^{\star}\right)$ will be called a vertical differential 1-form.

- A field $U \in \Gamma(V)$ will be called a vertical vector field.

\subsection{The generalized almost complex structure on the twistor space}

Now, we will construct a generalized complex structure $\rrbracket$ on $Z$ following the construction of almost complex structures on classical twistor spaces $[5,9,10,40]$. We need to define an automorphism

$$
\mathbb{J}: \mathbb{T} Z \longrightarrow \mathbb{T} Z
$$

such that

$$
\mathbb{J}^{2}=-\mathrm{Id}_{\mathbb{T} Z}
$$

Let $S \in Z, X \in \mathbb{T}_{S} Z$. Given the splitting

$$
\mathbb{T}_{S} Z=\left(V_{S} \oplus V_{S}^{\star}\right) \oplus\left(H_{S} \oplus H_{S}^{\star}\right),
$$

let us write

$$
x=x^{v}+x^{h}
$$

where the super-indices ${ }^{v}$ and ${ }^{h}$ denote vertical and horizontal parts of the generalized tangent bundle, i.e. $X^{v} \in V_{S} \oplus V_{S}^{\star}$ and $X^{h} \in H_{S} \oplus H_{S}^{\star}$. 
First, let us extend the complex structure $I$ of the fibre to $T Z$ and denote it by the same letter, i.e. for $U_{S} \in V_{S}, X_{S} \in H_{S}$

$$
\begin{aligned}
I_{S}: T_{S} Z & \rightarrow T_{S} Z \\
I_{S}\left(U_{S}\right) & =S \cdot U_{S}, \\
I_{S}\left(X_{S}\right) & =0,
\end{aligned}
$$

so that, by definition, for any differential 1-form $\beta$, the form $I^{\star} \beta$ vanishes on horizontal vectors (i.e. $I^{\star} \beta$ is vertical). Now, let $\alpha_{S} \in H_{S}^{\star}, \eta_{S} \in V_{S}^{\star}$ and define

$$
\begin{aligned}
\mathbb{J}_{S}: \mathbb{T}_{S} Z & \rightarrow \mathbb{T}_{S} Z \\
\mathbb{J}_{S}\left(U_{S}\right) & =I_{S}\left(U_{S}\right)=S \cdot U_{S}, \\
\mathbb{J}_{S}\left(\eta_{S}\right) & =-I_{S}^{\star}\left(\eta_{S}\right)=-\eta_{S} \circ I_{S}, \\
\mathbb{J}_{S}\left(X_{S}+\alpha_{S}\right) & =\widehat{d \pi}_{S}^{-1}\left(\varphi(S)\left(\widehat{d \pi}_{S}\left(X_{S}+\alpha_{S}\right)\right)\right) .
\end{aligned}
$$

Remark. Note that one can construct another generalized complex structure on $Z$ by considering $-I_{S}$ on the fibers.

\subsection{Useful lemmas}

Lemma 4.1. Let $X, Y$ be basic vector fields, $\alpha$ be a horizontal differential 1-form, $\eta$ a vertical 1-form, $U, W$ vertical vector fields and $f \in C^{\infty}(Z)$. Then

(a) $d \pi[X, Y]=[d \pi X, d \pi Y]$;

(b) $[X, U]$ is vertical;

(c) $\mathcal{L}_{X} \alpha$ is a horizontal differential 1-form;

(d) $\mathcal{L}_{U} \alpha$ is a horizontal differential 1-form;

(e) If $\alpha$ is basic, $\alpha=\pi^{\star}(\bar{\alpha})$ where $\bar{\alpha}$ is a differential 1 -form on $M$, then $\mathcal{L}_{U} \alpha=0$;

(f) $\mathcal{L}_{f X} \eta=f \mathcal{L}_{X} \eta$;

(g) the vertical part of the Lie bracket of two horizontal vector fields is tensorial, i.e. $[f X, Y]^{v}=f[X, Y]^{v}$.

Proof. Part (a) follows from [42, Prop. 8.2] since the fields on $Z$ are $\pi$-related to the fields on $M$. Part (b) follows from (a) since $U$ is $\pi$-related to 0 . Parts (c) - (g) follow from Cartan's formula.

From now on, given any vector field $W$, we will denote its local flow by $\phi_{t}^{W}$. Note that the flow $\phi_{t}^{X}$ of a basic vector field preserves the fibration structure of $Z$ and, since each point $S \in Z_{\pi(S)}$ is itself the complex structure of $T_{S} Z_{\pi(S)}, \phi_{t}^{X}$ also sends the complex structure $S$ of $T_{S} Z_{\pi(S)}$ to the complex structure $\phi_{t}^{X}(S)$ of $T_{\phi_{t}^{X}(S)} Z_{\pi(S)}$.

Lemma 4.2. Let $X$ be a basic vector field. Then

$$
\mathcal{L}_{X} I=0 \text {. }
$$

Lemma 4.3. Let $X$ be a basic vector field and $\eta$ a vertical differential 1-form. Then

$$
\left(\mathcal{L}_{X}(\eta \circ I)\right)^{v} \circ I=-\left(\mathcal{L}_{X} \eta\right)^{v}
$$

Proof. Let $Y$ be a basic vector field and $U$ a vertical vector field. Consider

$$
\begin{aligned}
\left(\left(\mathcal{L}_{X}(\eta \circ I)\right)^{v} \circ I\right)(Y+U) & =\left(\mathcal{L}_{X}(\eta \circ I)\right)^{v}(I(Y+U)) \\
& =\left(\mathcal{L}_{X}(\eta \circ I)\right)^{v}(I(U))
\end{aligned}
$$




$$
\begin{aligned}
& =X(\eta(I(I(U))))-\eta(I[X, I(U)]) \\
& =-X(\eta(U))-\eta\left(I\left(\mathcal{L}_{X}(I(U))\right)\right) \\
& =-X(\eta(U))-\eta\left(I\left(\left(\mathcal{L}_{X} I\right)(U)+I\left(\mathcal{L}_{X} U\right)\right)\right) \\
& =-X(\eta(U))-\eta\left(I\left(I\left(\mathcal{L}_{X} U\right)\right)\right) \\
& =-X(\eta(U))+\eta\left(\mathcal{L}_{X} U\right) \\
& =-X(\eta(U))+\eta([X, U]) \\
& =-\left(\mathcal{L}_{X} \eta\right)^{v}(Y+U),
\end{aligned}
$$

where we used Lemma 4.2.

Lemma 4.4. Let $\xi$ be vertical differential 1-form, $X, Y$ horizontal vector fields and $\beta$ a horizontal differential 1-form. Then

$$
\left\langle\mathbb{J}\left(\left(\mathcal{L}_{X} \xi\right)^{h}\right), Y+\beta\right\rangle=\frac{1}{2} \xi\left(\left[X,(\mathbb{J}(Y+\beta))_{v}\right]\right) .
$$

Proof. Consider $\left\{X_{1}, \ldots, X_{n}\right\} \cup\left\{\alpha_{1}, \ldots, \alpha_{n}\right\}$ be a local frame of $\mathbb{H}$ consisting of local basic vectors and forms such that

$$
\alpha_{i}\left(X_{j}\right)=\delta_{i j}
$$

Thus, $\left\{X_{1}+\alpha_{1}, \ldots, X_{n}+\alpha_{n}\right\} \cup\left\{X_{1}-\alpha_{1}, \ldots, X_{n}-\alpha_{n}\right\}$ is an orthonormal frame of $\mathbb{H}$, i.e.

$$
\begin{aligned}
\left\langle X_{i}+\alpha_{i}, X_{j}+\alpha_{j}\right\rangle & =1, \\
\left\langle X_{i}+\alpha_{i}, X_{j}-\alpha_{j}\right\rangle & =0, \\
\left\langle X_{i}-\alpha_{i}, X_{j}-\alpha_{j}\right\rangle & =-1 .
\end{aligned}
$$

Now,

$$
\begin{aligned}
\mathbb{J}\left(\left(\mathcal{L}_{X} \xi\right)^{h}\right)= & \sum_{i}\left\langle\mathbb{J}\left(\left(\mathcal{L}_{X} \xi\right)^{h}\right), X_{i}+\alpha_{i}\right\rangle\left(X_{i}+\alpha_{i}\right)-\left\langle\mathbb{J}\left(\left(\mathcal{L}_{X} \xi\right)^{h}\right), X_{i}-\alpha_{i}\right\rangle\left(X_{i}-\alpha_{i}\right) \\
= & -\sum_{i}\left\langle\left(\mathcal{L}_{X} \xi\right)^{h}, \mathbb{J} X_{i}+\mathbb{J} \alpha_{i}\right\rangle\left(X_{i}+\alpha_{i}\right)-\left\langle\left(\mathcal{L}_{X} \xi\right)^{h}, \mathbb{J} X_{i}-\mathbb{J} \alpha_{i}\right\rangle\left(X_{i}-\alpha_{i}\right) \\
= & -\sum_{i}\left(\left\langle\left(\mathcal{L}_{X} \xi\right)^{h}, \mathbb{J} X_{i}\right\rangle X_{i}+\left\langle\left(\mathcal{L}_{X} \xi\right)^{h}, \mathbb{J} X_{i}\right\rangle \alpha_{i}+\left\langle\left(\mathcal{L}_{X} \xi\right)^{h}, \mathbb{J} \alpha_{i}\right\rangle X_{i}+\left\langle\left(\mathcal{L}_{X} \xi\right)^{h}, \mathbb{J} \alpha_{i}\right\rangle \alpha_{i}\right. \\
& \left.-\left\langle\left(\mathcal{L}_{X} \xi\right)^{h}, \mathbb{J} X_{i}\right\rangle X_{i}+\left\langle\left(\mathcal{L}_{X} \xi\right)^{h}, \mathbb{J} X_{i}\right\rangle \alpha_{i}+\left\langle\left(\mathcal{L}_{X} \xi\right)^{h}, \mathbb{J} \alpha_{i}\right\rangle X_{i}-\left\langle\left(\mathcal{L}_{X} \xi\right)^{h}, \mathbb{J} \alpha_{i}\right\rangle \alpha_{i}\right) \\
= & -2 \sum_{i}\left\langle\left(\mathcal{L}_{X} \xi\right)^{h}, \mathbb{J} X_{i}\right\rangle \alpha_{i}+\left\langle\left(\mathcal{L}_{X} \xi\right)^{h}, \mathbb{J} \alpha_{i}\right\rangle X_{i} \\
= & -\sum_{i} \mathcal{L}_{X} \xi\left(\left(\mathbb{J} X_{i}\right)_{v}\right) \alpha_{i}+\mathcal{L}_{X} \xi\left(\left(\mathbb{J} \alpha_{i}\right)_{v}\right) X_{i} \\
= & -\sum_{i} X \xi\left(\left(\mathbb{J} X_{i}\right)_{v}\right)-\xi\left(\left[X,\left(\mathbb{J} X_{i}\right)_{v}\right]\right) \alpha_{i}+X \xi\left(\left(\mathbb{J} \alpha_{i}\right)_{v}\right)-\xi\left(\left[X,\left(\mathbb{J} \alpha_{i}\right)_{v}\right]\right) X_{i} . \\
= & \sum_{i} \xi\left(\left[X,\left(\mathbb{J} X_{i}\right)_{v}\right]\right) \alpha_{i}+\xi\left(\left[X,\left(\mathbb{J} \alpha_{i}\right)_{v}\right]\right) X_{i} .
\end{aligned}
$$

Now, let us pair it with $Y+\beta$

$$
\begin{aligned}
\left\langle\mathbb{J}\left(\left(\mathcal{L}_{X} \xi\right)^{h}\right), Y+\beta\right\rangle & =\sum_{i}\left\langle\xi\left(\left[X,\left(\mathbb{J} X_{i}\right)_{v}\right]\right) \alpha_{i}+\xi\left(\left[X,\left(\mathbb{J} \alpha_{i}\right)_{v}\right]\right) X_{i}, Y+\beta\right\rangle \\
& =\frac{1}{2} \sum_{i} \xi\left(\left[X,\left(\mathbb{J} X_{i}\right)_{v}\right]\right) \alpha_{i}(Y)+\xi\left(\left[X,\left(\mathbb{J} \alpha_{i}\right)_{v}\right]\right) \beta\left(X_{i}\right) \\
& =\frac{1}{2} \sum_{i} \xi\left(\left[X,\left(\mathbb{J} X_{i}\right)_{v}\right]^{v}\right) \alpha_{i}(Y)+\xi\left(\left[X,\left(\mathbb{J} \alpha_{i}\right)_{v}\right]^{v}\right) \beta\left(X_{i}\right)
\end{aligned}
$$




$$
\begin{aligned}
& =\frac{1}{2} \sum_{i} \xi\left(\left[X, \alpha_{i}(Y)\left(\mathbb{J} X_{i}\right)_{v}\right]^{v}\right)+\xi\left(\left[X, \beta\left(X_{i}\right)\left(\mathbb{J} \alpha_{i}\right)_{v}\right]^{v}\right) \\
& =\frac{1}{2} \sum_{i} \xi\left(\left[X,\left(\mathbb{J}\left(\alpha_{i}(Y) X_{i}\right)\right)_{v}\right]\right)+\xi\left(\left[X,\left(\mathbb{J}\left(\beta\left(X_{i}\right) \alpha_{i}\right)\right)_{v}\right]\right) \\
& =\frac{1}{2} \xi\left(\left[X,\left(\mathbb{J}\left(\sum_{i} \alpha_{i}(Y) X_{i}\right)\right)_{v}\right]\right)+\xi\left(\left[X,\left(\mathbb{J}\left(\sum_{i} \beta\left(X_{i}\right) \alpha_{i}\right)\right){ }_{v}\right]\right) \\
& =\frac{1}{2} \xi\left(\left[X,(\mathbb{J} Y)_{v}\right]\right)+\xi\left(\left[X,(\mathbb{J} \beta)_{v}\right]\right) \\
& =\frac{1}{2} \xi\left(\left[X,(\mathbb{J} Y)_{v}\right]+\left[X,(\mathbb{J} \beta)_{v}\right]\right) \\
& =\frac{1}{2} \xi\left(\left[X,(\mathbb{J} Y)_{v}+(\mathbb{J} \beta)_{v}\right]\right) \\
& =\frac{1}{2} \xi\left(\left[X,(\mathbb{J} Y+\mathbb{J} \beta)_{v}\right]\right) \\
& =\frac{1}{2} \xi\left(\left[X,(\mathbb{J}(Y+\beta))_{v}\right]\right),
\end{aligned}
$$

where we used Lemma 4.1(g).

Lemma 4.5. Let $R$ be the curvature tenrsor of the induced connection on $\operatorname{End}(\mathbb{T} M), S_{0} \in Z, x_{0}=\pi\left(S_{0}\right)$, $X_{S_{0}}, Y_{S_{0}} \in H_{S_{0}}, \bar{X}_{x_{0}}=d \pi_{S_{0}} X_{S_{0}}, \bar{Y}_{x_{0}}=d \pi_{S_{0}} Y_{S_{0}}$. Then

$$
R\left(\bar{X}_{\chi_{0}}, \bar{Y}_{\chi_{0}}\right)\left(S_{0}\right)=-[X, Y]_{S_{0}}^{v}
$$

where $X$ and $Y$ are any local horizontal extensions of $X_{S_{0}}$ and $Y_{S_{0}}$.

Proof. This follows from [42, Thm. 3.2, p. 118] applied to the bundle End(TM) with its induced connection as an associated bundle of $P_{G_{r}}$.

\section{Integrability of $\mathbb{J}$}

Let $S_{0} \in Z, x_{0}=\pi\left(S_{0}\right) \in M$ and $\bar{x}, \bar{y}, \bar{z} \in \Gamma(\mathbb{T} M)$. We will consider the following two conditions on the curvature and generalized torsion of the connection on $\mathbb{T} M$ :

$$
\begin{aligned}
0= & R\left(\left(\varphi\left(S_{0}\right) \bar{x}_{x_{0}}\right)_{v},\left(\varphi\left(S_{0}\right) \bar{y}_{x_{0}}\right)_{v}\right)\left(S_{0}\right)-S_{0} \cdot R\left(\left(\varphi\left(S_{0}\right) \bar{x}_{x_{0}}\right)_{v},\left(\bar{y}_{x_{0}}\right)_{v}\right)\left(S_{0}\right) \\
& -S_{0} \cdot R\left(\left(\varphi\left(S_{0}\right) \bar{x}_{x_{0}}\right)_{v},\left(\varphi\left(S_{0}\right) \bar{y}_{x_{0}}\right)_{v}\right)\left(S_{0}\right)-R\left(\left(\bar{x}_{x_{0}}\right)_{v},\left(\bar{y}_{x_{0}}\right)_{v}\right)\left(S_{0}\right) \\
0= & \mathcal{T}\left(\bar{x}_{x_{0}}, \bar{y}_{x_{0}}, \bar{z}_{x_{0}}\right)-\mathcal{T}\left(\varphi\left(S_{0}\right) \bar{x}_{x_{0}}, \varphi\left(S_{0}\right) \bar{y}_{x_{0}}, \bar{z}_{x_{0}}\right) \\
& -\mathcal{T}\left(\varphi\left(S_{0}\right) \bar{x}_{x_{0}}, \bar{y}_{x_{0}}, \varphi\left(S_{0}\right) \bar{z}_{x_{0}}\right)-\mathcal{T}\left(\bar{x}_{x_{0}}, \varphi\left(S_{0}\right) \bar{y}_{x_{0}}, \varphi\left(S_{0}\right) \bar{z}_{x_{0}}\right)
\end{aligned}
$$

Theorem 5.1. Let $M$ be a generalized almost even-Clifford manifold endowed with a connection on its reduced structure bundle $P_{G_{r}}, Z$ its twistor space and $\mathbb{J}$ its connection-dependent generalized almost complex structure. If the curvature and the generalized torsion satisfy conditions (1) and (2) respectively, then $\mathbb{J}$ is integrable,

$$
\mathcal{N}^{\mathbb{J}}=0 \text {. }
$$

Remark. Note that condition (1) is not invariant under a change of sign of the complex structure on the fiber.

We will split the proof of the theorem in the following three lemmas.

\section{Lemma 5.1.}

$$
\left.\mathcal{N}^{\mathbb{J}}\right|_{\mathbb{V} \times \mathbb{V}} \equiv 0
$$


Lemma 5.2. If the curvature satisfies conditions (1), then

$$
\left.\mathcal{N}^{\mathbb{J}}\right|_{\mathbb{V} \times \mathbb{H}} \equiv 0 \text {. }
$$

Lemma 5.3. If the curvature and the generalized torsion satisfy conditions (1) and (2) respectively, then

$$
\left.\mathcal{N}^{\mathbb{J}}\right|_{\mathbb{H} \times \mathbb{H}} \equiv 0 .
$$

Before proving the theorem, let us consider the following example.

Example. A manifold with a parallel even-Clifford structure is a Riemannian manifold $M$ such that the almost even-Clifford structure is parallel with respect to the Levi-Civita connection [31]. The even-Clifford structure $\left\{J_{i j}\right\}$ gives rise to a generalized almost even-Clifford structure $\left\{J_{i j} \oplus\left(-J_{i j}^{\star}\right)\right\}$ on $M$. Since the curvature tensor and the local generalized almost complex structures are diagonal on $T M \oplus T^{*} M$, condition (1) reduces to the curvature condition for the integrability of the complex structure on the classical twistor space of $M$ (in the notation of [5])

$$
\left[R_{S X, S Y}, S\right]-S\left[R_{S X, Y}, S\right]-S\left[R_{X, S Y}, S\right]-\left[R_{X, Y}, S\right]=0,
$$

which parallel even Clifford manifolds satisfy [5, Lemma 3.2]. Since the Levi-Civita connection is torsion free, the generalized torsion of the induced connection on $\mathbb{T} M$ is zero, and condition (2) is also fulfilled. Thus, the twistor space of the induced generalized almost even-Clifford structure of a parallel even-Clifford manifold admits an integrable generalized complex structure, which is consistent with the integrability of the complex structure of its classical twistor space.

\subsection{Proof of Lemma 5.1}

Let $U, V \in \Gamma(V), \eta, \xi \in \Gamma\left(V^{\star}\right)$. Then,

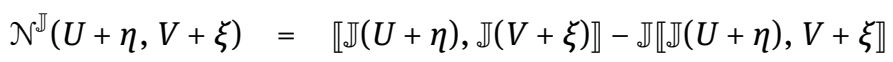

$$
\begin{aligned}
& -\mathbb{J} \llbracket U+\eta, \mathbb{J}(V+\xi) \rrbracket-\llbracket U+\eta, V+\xi \rrbracket \\
& =\llbracket I(U)-I^{\star}(\eta), I(V)-I^{\star}(\xi) \rrbracket-\mathbb{J} \llbracket I(U)-I^{\star}(\eta), V+\xi \rrbracket \\
& -J \llbracket U+\eta, I(V)-I^{\star}(\xi) \rrbracket-\llbracket U+\eta, V+\xi \rrbracket \\
& =N^{I}(U, V)+\theta_{U, V} \\
& =\theta_{U, V} \text {. }
\end{aligned}
$$

where we have used the integrability of $I$ on the fiber, and

$$
\begin{aligned}
\theta_{U, V}:= & \mathcal{L}_{I U}\left(-I^{\star} \eta\right)-\mathcal{L}_{I V}\left(-I^{\star} \xi\right)-\frac{1}{2} d\left(i_{I U}\left(-I^{\star} \eta\right)-i_{I Y}\left(-I^{\star} \xi\right)\right) \\
& +I^{\star}\left(\mathcal{L}_{I U} \eta-\mathcal{L}_{V}\left(-I^{\star} \xi\right)-\frac{1}{2} d\left(i_{I U} \eta-i_{V}\left(-I^{\star} \xi\right)\right)\right) \\
& +I^{\star}\left(\mathcal{L}_{U}\left(-I^{\star} \eta\right)-\mathcal{L}_{I V} \xi-\frac{1}{2} d\left(i_{U}\left(-I^{\star} \eta\right)-i_{I V} \xi\right)\right) \\
& -\left(\mathcal{L}_{U} \eta-\mathcal{L}_{V} \xi-\frac{1}{2} d\left(i_{U} \eta-i_{V} \xi\right)\right) .
\end{aligned}
$$

First notice

$$
-\frac{1}{2} d\left(i_{I U}\left(-I^{\star} \eta\right)-i_{I V}\left(-I^{\star} \xi\right)\right)+I^{\star}\left(-\frac{1}{2} d\left(i_{I U} \eta-i_{V}\left(-I^{\star} \xi\right)\right)\right)+I^{\star}\left(-\frac{1}{2} d\left(i_{U}\left(-I^{\star} \eta\right)-i_{I V} \xi\right)\right)+\frac{1}{2} d\left(i_{U} \eta-i_{V} \xi\right)=0,
$$

so that

$$
\theta_{U, V}=\mathcal{L}_{I U}\left(-I^{\star} \eta\right)-\mathcal{L}_{I V}\left(-I^{\star} \xi\right)+I^{\star}\left(\mathcal{L}_{I U} \eta-\mathcal{L}_{V}\left(-I^{\star} \xi\right)\right)
$$




$$
+I^{\star}\left(\mathcal{L}_{U}\left(-I^{\star} \eta\right)-\mathcal{L}_{I V} \xi\right)-\left(\mathcal{L}_{U} \eta-\mathcal{L}_{V} \xi\right),
$$

Evaluating this 1-form on $W \in \Gamma(V)$,

$$
\begin{aligned}
\theta_{U, V}(W)= & \mathcal{L}_{I U}\left(-I^{\star} \eta\right)(W)-\mathcal{L}_{I V}\left(-I^{\star} \xi\right)(W)+I^{\star}\left(\mathcal{L}_{I U} \eta-\mathcal{L}_{V}\left(-I^{\star} \xi\right)\right)(W) \\
& +I^{\star}\left(\mathcal{L}_{U}\left(-I^{\star} \eta\right)-\mathcal{L}_{I V} \xi\right)(W)-\left(\mathcal{L}_{U} \eta(W)-\mathcal{L}_{V} \xi(W)\right) \\
= & (I U)\left(-I^{\star} \eta(W)\right)+I^{\star} \eta([I U, W])-(I V)\left(-I^{\star} \xi(W)\right)+I^{\star} \xi([I V, W]) \\
& +(I U)(\eta(-I W))-\eta([I U,-I W])-V\left(-I^{\star} \xi(-I W)\right)-I^{\star} \xi([V,-I W]) \\
& +U\left(-I^{\star} \eta(-I W)\right)+I^{\star} \eta([U,-I W])-(I V)(\xi(-I W))+\xi([I V,-I W]) \\
& -U(\eta(W))+\eta([U, W])+V(\xi(W))-\xi([V, W]) \\
= & \eta\left(-N^{I}(U, W)\right)+\xi\left(N^{I}(V, W)\right) \\
= & 0,
\end{aligned}
$$

which proves Lemma 5.1.

\subsection{Proof of Lemma 5.2}

Let $S_{0} \in Z, U+\eta \in \Gamma(\mathbb{V}), X+\alpha \in \Gamma(\mathbb{H})$. Consider

$$
\begin{aligned}
& \mathcal{N}^{\mathbb{J}}(U+\eta, X+\alpha)_{S_{0}}=\llbracket \mathbb{J}(U+\eta), \mathbb{J}(X+\alpha) \rrbracket s_{S_{0}}-\mathbb{J} \llbracket \mathbb{J}(U+\eta), X+\alpha \rrbracket S_{0} \\
& -\mathbb{\amalg} \llbracket U+\eta, \mathbb{J}(X+\alpha) \rrbracket_{S_{0}}-\llbracket U+\eta, X+\alpha \rrbracket_{S_{0}}
\end{aligned}
$$

We will examine one term at a time. The first term is

$$
\begin{aligned}
\llbracket \mathbb{J}(U+\eta), \mathbb{J}(X+\alpha) \rrbracket= & \llbracket I(U)-I^{\star}(\eta), \mathbb{J}(X+\alpha) \rrbracket \\
= & {\left[I(U),(\mathbb{J}(X+\alpha))_{v}\right]+\mathcal{L}_{I(U)}\left((\mathbb{J}(X+\alpha))_{f}\right)-\frac{1}{2} d\left((\mathbb{J}(X+\alpha))_{f}(I(U))\right) } \\
& +\mathcal{L}_{(\mathbb{J}(X+\alpha))_{v}} I^{\star}(\eta)-\frac{1}{2} d\left(I^{\star}(\eta)\left((\mathbb{J}(X+\alpha))_{v}\right)\right) \\
= & {\left[I(U),(\mathbb{J}(X+\alpha))_{v}\right]+\mathcal{L}_{I(U)}(\mathbb{J}(X+\alpha))_{f}+\mathcal{L}_{(\mathbb{J}(X+\alpha))_{v}}(\eta \circ I) } \\
= & {\left[I(U),(\mathbb{J}(X+\alpha))_{v}\right]^{v}+\left[I(U),(\mathbb{J}(X+\alpha))_{v}\right]^{h}+\mathcal{L}_{I(U)}(\mathbb{J}(X+\alpha))_{f} } \\
& +\left(\mathcal{L}_{(\mathbb{J}(X+\alpha))_{v}}(\eta \circ I)\right)^{v}+\left(\mathcal{L}_{(\mathbb{J}(X+\alpha))_{v}}(\eta \circ I)\right)^{h} .
\end{aligned}
$$

The second term is

$$
\begin{aligned}
-\mathbb{J} \llbracket \mathbb{J}(U+\eta), X+\alpha \rrbracket & =-\mathbb{J}\left([I(U), X]+\mathcal{L}_{I(U)} \alpha-\mathcal{L}_{X}\left(-I^{\star}(\eta)\right)-\frac{1}{2} d\left(\alpha(I(U))-\left(-I^{\star}(\eta)\right)(X)\right)\right) \\
& \left.=-\mathbb{J}\left([I(U), X]+\mathcal{L}_{X}(\eta \circ I)+\eta(I(X))\right)\right) \\
& =-\mathbb{J}\left([I(U), X]+\mathcal{L}_{X}(\eta \circ I)\right) \\
& =-\mathbb{J}\left(\left(\mathcal{L}_{X} I\right)(U)-I[X, U]+\left(\mathcal{L}_{X}(\eta \circ I)\right)^{v}+\left(\mathcal{L}_{X}(\eta \circ I)\right)^{h}\right) \\
& =-\mathbb{J}\left(-I[X, U]+\left(\mathcal{L}_{X}(\eta \circ I)\right)^{v}+\left(\mathcal{L}_{X}(\eta \circ I)\right)^{h}\right) \\
& =-\mathbb{J}\left(-I[X, U]+\left(\mathcal{L}_{X}(\eta \circ I)\right)^{v}\right)-\mathbb{J}\left(\left(\mathcal{L}_{X}(\eta \circ I)\right)^{h}\right) .
\end{aligned}
$$

The third term is

$$
-\mathbb{J} \llbracket U+\eta, \mathbb{J}(X+\alpha) \rrbracket=-\mathbb{J}\left(\left[U,(\mathbb{J}(X+\alpha))_{v}\right]+\mathcal{L}_{U}(\mathbb{J}(X+\alpha))_{f}-\mathcal{L}_{(\mathbb{J}(X+\alpha))_{v}} \eta\right.
$$




$$
\begin{aligned}
& \left.-\frac{1}{2} d\left((\mathbb{J}(X+\alpha))_{f}(U)-\eta\left((\mathbb{J}(X+\alpha))_{v}\right)\right)\right) \\
= & -\mathbb{J}\left(\left[U,(\mathbb{J}(X+\alpha))_{v}\right]+\mathcal{L}_{U}(\mathbb{J}(X+\alpha))_{f}-\mathcal{L}_{(\mathbb{J}(X+\alpha))_{v}} \eta\right) \\
= & -\mathbb{J}\left(\left[U,(\mathbb{J}(X+\alpha))_{v}\right]^{v}+\left[U,(\mathbb{J}(X+\alpha))_{v}\right]^{h}+\mathcal{L}_{U}(\mathbb{J}(X+\alpha))_{f}\right. \\
& \left.-\left(\mathcal{L}_{(\mathbb{J}(X+\alpha))_{v}} \eta\right)^{v}-\left(\mathcal{L}_{(\mathbb{J}(X+\alpha))_{v}} \eta\right)^{h}\right) \\
= & -\mathbb{J}\left(\left[U,(\mathbb{J}(X+\alpha))_{v}\right]^{v}-\left(\mathcal{L}_{(\mathbb{J}(X+\alpha))_{v}} \eta\right)^{v}\right. \\
& \left.\left.+\left[U,(\mathbb{J}(X+\alpha))_{v}\right]^{h}+\mathcal{L}_{U}(\mathbb{J}(X+\alpha))_{f}\right)-\left(\mathcal{L}_{(\mathbb{J}(X+\alpha))_{v}} \eta\right)^{h}\right) \\
= & -I\left[U,(\mathbb{J}(X+\alpha))_{v}\right]^{v}-\left(\mathcal{L}_{(\mathbb{J}(X+\alpha))_{v}} \eta\right)^{v} \circ I \\
& -\mathbb{J}\left(\left[U,(\mathbb{J}(X+\alpha))_{v}\right]^{h}+\mathcal{L}_{U}(\mathbb{J}(X+\alpha))_{f}\right)+\left(\mathbb{J}\left(\left(\mathcal{L}_{(\mathbb{J}(X+\alpha))_{v}} \eta\right)^{h}\right)\right)_{f} .
\end{aligned}
$$

The fourth term is

$$
\begin{aligned}
-\llbracket U+\eta, X+\alpha \rrbracket & =-\left([U, X]+\left(\mathcal{L}_{U} \alpha\right)-\left(\mathcal{L}_{X} \eta\right)-\frac{1}{2} d(\alpha(U))+\frac{1}{2} d(\eta(X))\right) \\
& =[X, U]+\mathcal{L}_{X} \eta \\
& =[X, U]+\left(\mathcal{L}_{X} \eta\right)^{v}+\left(\mathcal{L}_{X} \eta\right)^{h} .
\end{aligned}
$$

Claim 1.

$$
-\mathbb{J}\left(-I[X, U]+\left(\mathcal{L}_{X}(\eta \circ I)\right)^{v}\right)+[X, U]+\left(\mathcal{L}_{X} \eta\right)^{v}=0 .
$$

Using Lemma 4.3

$$
\begin{aligned}
-\mathbb{J}\left(-I[X, U]+\left(\mathcal{L}_{X}(\eta \circ I)\right)^{v}\right) & =I(I[X, U])+\left(\mathcal{L}_{X}(\eta \circ I)\right)^{v} \circ I \\
& =-[X, U]+\left(\mathcal{L}_{X}(\eta \circ I)\right)^{v} \circ I \\
& =-[X, U]-\left(\mathcal{L}_{X} \eta\right)^{v} .
\end{aligned}
$$

Claim 2.

$$
\left(\mathcal{L}_{(\mathbb{J}(X+\alpha))_{v}}\left(\eta \circ I_{z}\right)\right)^{v}-\left(\mathcal{L}_{(\mathbb{J}(X+\alpha))_{v}} \eta\right)^{v} \circ I=0 .
$$

Let us write

$$
(\mathbb{J}(X+\alpha))_{v}=\sum_{i} a_{i} X_{i}
$$

in terms of a basis $\left\{X_{i}\right\}$ of local basic vector fields. Then, using Lemmas 4.1 and 4.2

$$
\begin{aligned}
\left(\left(\mathcal{L}_{(\mathbb{J}(X+\alpha))_{v}} \eta\right)^{v} \circ I\right)(Y+U) & =\left(\left(\mathcal{L}_{(\mathbb{J}(X+\alpha))_{v}} \eta\right)^{v}\right)(I(Y+U)) \\
& =\left(\mathcal{L}_{(\mathbb{J}(X+\alpha))_{v}} \eta\right)(I(U)) \\
& =\left(\mathcal{L}_{\sum_{i} a_{i} X_{i}} \eta\right)(I(U)) \\
& =\left(\sum_{i} a_{i} \mathcal{L}_{X_{i}} \eta\right)(I(U)) \\
& =\sum_{i} a_{i}\left(\mathcal{L}_{X_{i}} \eta\right)(I(U)) \\
& =\sum_{i} a_{i}\left(X_{i}\left(\eta(I(U))-\eta\left(\left[X_{i}, I(U)\right]\right)\right)\right. \\
& =\sum_{i} a_{i}\left(X_{i}\left(\eta(I(U))-\eta\left(\left(\mathcal{L}_{X_{i}} I\right)(U)+I\left[X_{i}, U\right]\right)\right)\right. \\
& =\sum_{i} a_{i}\left(X_{i}\left(\eta(I(U))-\eta\left(I\left[X_{i}, U\right]\right)\right),\right.
\end{aligned}
$$




$$
\begin{aligned}
\left(\mathcal{L}_{(\mathbb{J}(X+\alpha))_{v}}\left(\eta \circ I_{z}\right)\right)^{v}(Y+U) & =\left(\mathcal{L}_{(\mathbb{J}(X+\alpha))_{v}}\left(\eta \circ I_{z}\right)\right)(U) \\
& =\sum_{i} a_{i}\left(\mathcal{L}_{X_{i}}(\eta \circ I)\right)(U) \\
& =\sum_{i} a_{i}\left(X_{i}(\eta(I(U)))-\eta\left(I\left[X_{i}, U\right]\right)\right) .
\end{aligned}
$$

Claim 3.

$$
\left[I(U),(\mathbb{J}(X+\alpha))_{V}\right]^{v}-I\left[U,(\mathbb{J}(X+\alpha))_{V}\right]^{v}=0 .
$$

Indeed, we have

$$
\left[I(U),(\mathbb{J}(X+\alpha))_{v}\right]=\sum_{i} d a_{i}(I(U)) X_{i}+\sum_{i} a_{i}\left[I(U), X_{i}\right]
$$

so that

$$
\left(\left[I(U),(\mathbb{J}(X+\alpha))_{v}\right]\right)^{v}=\sum_{i} a_{i}\left[I(U), X_{i}\right] .
$$

Similarly

$$
\left[U,(\mathbb{J}(X+\alpha))_{v}\right]=\sum_{i} d a_{i}(U) X_{i}+\sum_{i} a_{i}\left[U, X_{i}\right]
$$

and

$$
\mathbb{J}\left[U,(\mathbb{J}(X+\alpha))_{v}\right]=\mathbb{J}\left(\sum_{i} d a_{i}(U) X_{i}\right)+\sum_{i} a_{i} I\left[U, X_{i}\right]
$$

Thus

$$
\begin{aligned}
\mathbb{J}\left(\left(\left[U,(\mathbb{J}(X+\alpha))_{v}\right]\right)^{v}\right) & =\sum_{i} a_{i} I\left[U, X_{i}\right] \\
& =\left(\mathbb{J}\left[U,(\mathbb{J}(X+\alpha))_{v}\right]\right)^{v},
\end{aligned}
$$

and

$$
\begin{aligned}
\left(\left[I(U),(\mathbb{J}(X+\alpha))_{v}\right]-\mathbb{J}\left[U,(\mathbb{J}(X+\alpha))_{v}\right]\right)^{v} & =\left(\left[I(U),(\mathbb{J}(X+\alpha))_{v}\right]\right)^{v}-\left(\mathbb{J}\left[U,(\mathbb{J}(X+\alpha))_{v}\right]\right)^{v} \\
& =\sum_{i} a_{i}\left[I(U), X_{i}\right]-\sum_{i} a_{i} I\left[U, X_{i}\right] \\
& =\sum_{i} a_{i}\left(\left[I(U), X_{i}\right]-I\left[U, X_{i}\right]\right) \\
& =\sum_{i} a_{i}\left(-\mathcal{L}_{X_{i}}(I(U))+I\left(\mathcal{L}_{X_{i}} U\right)\right) \\
& =\sum_{i} a_{i}\left(-\left(\mathcal{L}_{X_{i}} I\right)(U)-I\left(\mathcal{L}_{X_{i}} U\right)+I\left(\mathcal{L}_{X_{i}} U\right)\right) \\
& =0 .
\end{aligned}
$$

Claim 4.

$$
\left[I(U),(\mathbb{J}(X+\alpha))_{V}\right]^{h}-\mathbb{J}\left(\left[U,(\mathbb{J}(X+\alpha))_{V}\right]^{h}\right)=0 .
$$

Consider

$$
d \pi_{S_{0}}\left(\left[U,(\mathbb{J}(X+\alpha))_{v}\right]_{S_{0}}\right)=d \pi_{S_{0}}\left(\mathcal{L}_{U}(\mathbb{J}(X+\alpha))_{v}\right)_{S_{0}}
$$




$$
\begin{aligned}
& =d \pi_{S_{0}}\left(\operatorname { l i m } _ { t \rightarrow 0 } \frac { 1 } { t } \left(d\left(\phi_{-t}^{U}\right)_{\phi_{t}^{U}\left(S_{0}\right)}\left(\widehat{d \pi}_{\phi_{t}^{U}\left(S_{0}\right)}^{-1} \varphi\left(\phi_{t}^{U}\left(S_{0}\right)\right) \widehat{d \pi}_{\phi_{t}^{U}\left(S_{0}\right)}\left(X_{\phi_{t}^{U}\left(S_{0}\right)}+\alpha_{\phi_{t}^{U}\left(S_{0}\right)}\right)\right)_{v}\right.\right. \\
& \left.\left.-\left(\widehat{d \pi}_{S_{0}}^{-1} \varphi\left(S_{0}\right) \widehat{d \pi}_{S_{0}}\left(X_{S_{0}}+\alpha_{S_{0}}\right)\right)_{V}\right)\right) \\
& =d \pi_{S_{0}}\left(\operatorname { l i m } _ { t \rightarrow 0 } \frac { 1 } { t } \left(d\left(\phi_{-t}^{U}\right)_{\phi_{t}^{U}\left(S_{0}\right)}\left(\widehat{d \pi}_{\phi_{t}^{U}\left(S_{0}\right)}^{-1} \varphi\left(\phi_{t}^{U}\left(S_{0}\right)\right)\left(\bar{X}_{x_{0}}+\bar{\alpha}_{x_{0}}\right)\right)_{v}\right.\right. \\
& \left.\left.-\left(\widehat{d \pi}_{S_{0}}^{-1} \varphi\left(S_{0}\right)\left(\bar{X}_{x_{0}}+\bar{\alpha}_{x_{0}}\right)\right)_{v}\right)\right) \\
& =\lim _{t \rightarrow 0} \frac{1}{t}\left(d \pi_{\phi_{t}^{U}\left(S_{0}\right)}\left(\widehat{d \pi}_{\phi_{t}^{U}\left(S_{0}\right)}^{-1} \varphi\left(\phi_{t}^{U}\left(S_{0}\right)\right)\left(\bar{X}_{x_{0}}+\bar{\alpha}_{x_{0}}\right)\right)_{v}\right. \\
& \left.-d \pi_{S_{0}}\left(\widehat{d \pi}_{S_{0}}^{-1} \varphi\left(S_{0}\right)\left(\bar{X}_{x_{0}}+\bar{\alpha}_{x_{0}}\right)\right)_{v}\right) \\
& =\lim _{t \rightarrow 0} \frac{1}{t}\left(\left(\widehat{d \pi}_{\phi_{t}^{U}\left(S_{0}\right)} \widehat{d \pi}_{\phi_{t}^{U}\left(S_{0}\right)}^{-1} \varphi\left(\phi_{t}^{U}\left(S_{0}\right)\right)\left(\bar{X}_{x_{0}}+\bar{\alpha}_{x_{0}}\right)\right)_{v}\right. \\
& \left.-\left(\widehat{d \pi}_{S_{0}} \widehat{d \pi}_{S_{0}}^{-1} \varphi\left(S_{0}\right)\left(\bar{X}_{x_{0}}+\bar{\alpha}_{x_{0}}\right)\right)_{v}\right) \\
& =\lim _{t \rightarrow 0} \frac{1}{t}\left(\left(\varphi\left(\phi_{t}^{U}\left(S_{0}\right)\right)\left(\bar{X}_{x_{0}}+\bar{\alpha}_{x_{0}}\right)\right)_{v}-\left(\varphi\left(S_{0}\right)\left(\bar{X}_{x_{0}}+\bar{\alpha}_{x_{0}}\right)\right)_{v}\right) \\
& =\left(\left(\lim _{t \rightarrow 0} \frac{1}{t}\left(\varphi\left(\phi_{t}^{U}\left(S_{0}\right)\right)-\varphi\left(S_{0}\right)\right)\right)\left(\bar{X}_{x_{0}}+\bar{\alpha}_{x_{0}}\right)\right)_{v} \\
& =\left(\varphi\left(\lim _{t \rightarrow 0} \frac{1}{t}\left(\phi_{t}^{U}\left(S_{0}\right)-S_{0}\right)\left(\bar{X}_{x_{0}}+\bar{\alpha}_{x_{0}}\right)\right)_{v}\right. \\
& =\left(\varphi\left(U_{S_{0}}\right)\left(\bar{X}_{x_{0}}+\bar{\alpha}_{x_{0}}\right)\right)_{v} \text {. }
\end{aligned}
$$

where we have used $\pi \circ \phi_{t}^{U}=\pi$. Moreover,

$$
\begin{aligned}
d \pi_{S_{0}}\left(\left[I(U),(\mathbb{J}(X+\alpha))_{v}\right]_{S_{0}}\right) & =\left(\varphi\left(I_{S_{0}} U_{S_{0}}\right)\left(\bar{X}_{x_{0}}+\bar{\alpha}_{x_{0}}\right)\right)_{v} \\
& =\left(\varphi\left(S_{0} \cdot U_{S_{0}}\right)\left(\bar{X}_{x_{0}}+\bar{\alpha}_{x_{0}}\right)\right)_{v},
\end{aligned}
$$

and

$$
\begin{aligned}
d \pi_{S_{0}} \mathbb{J}\left(\left[U,(\mathbb{J}(X+\alpha))_{v}\right]_{S_{0}}\right) & =d \pi_{S_{0}}\left({\widehat{d \pi_{S_{0}}}}^{-1} \varphi\left(S_{0}\right) \widehat{d \pi}_{S_{0}}\left(\left[U,(\mathbb{J}(X+\alpha))_{v}\right]_{S_{0}}\right)\right) \\
& =\left(\varphi\left(S_{0}\right) \varphi\left(U_{S_{0}}\right)\left(\bar{X}_{x_{0}}+\bar{\alpha}_{X_{0}}\right)\right)_{v} \\
& =\left(\varphi\left(S_{0} \cdot U_{S_{0}}\right)\left(\bar{X}_{x_{0}}+\bar{\alpha}_{x_{0}}\right)\right)_{v},
\end{aligned}
$$

where we have used that $\varphi$ is a fibre-wise algebra homomorphism.

\section{Claim 5.}

$$
\mathcal{L}_{I(U)}(\mathbb{J}(X+\alpha))_{f}-\mathbb{J}\left(\mathcal{L}_{U}(\mathbb{\mathbb { J }}(X+\alpha))_{f}\right)=0 .
$$

By Lemma 4.1, $\mathcal{L}_{U}(\mathbb{J}(X+\alpha))_{f}$ is horizontal. Hence, let us consider $A$ a basic vector field, $\bar{A}$ its projection to $M$ and

$$
\begin{aligned}
\widehat{d \pi}_{S_{0}}\left(\mathcal{L}_{U}(\mathbb{J}(X+\alpha))_{f}\right)_{S_{0}}\left(\bar{A}_{x_{0}}\right) & =\left(\mathcal{L}_{U}(\mathbb{J}(X+\alpha))_{f}\right)\left(A_{S_{0}}\right) \\
& =\left(U\left((\mathbb{J}(X+\alpha))_{f}(A)\right)\right)_{S_{0}}-\left((\mathbb{J}(X+\alpha))_{f}([U, A])\right)_{S_{0}} \\
& =\left(U\left((\mathbb{J}(X+\alpha))_{f}(A)\right)\right)_{S_{0}} \\
& =\left(d\left((\mathbb{J}(X+\alpha))_{f}(A)\right)_{S_{0}}\right)^{v}\left(U_{S_{0}}\right) \\
& =\left(d\left((\mathbb{J}(X+\alpha))_{f}(A)\right)_{S_{0}}\right)\left(U_{S_{0}}\right) \\
& =\lim _{t \rightarrow 0} \frac{1}{t}\left(\left((\mathbb{J}(X+\alpha))_{f}(A)\right)_{\phi_{t}^{U}\left(S_{0}\right)}-\left((\mathbb{J}(X+\alpha))_{f}(A)\right)_{S_{0}}\right) \\
& =\lim _{t \rightarrow 0} \frac{1}{t}\left(\left(\left(\widehat{d \pi}_{\phi_{t}^{U}\left(S_{0}\right)}^{-1} \varphi\left(\phi_{t}^{U}\left(S_{0}\right)\right) \widehat{d \pi}_{\phi_{t}^{U}\left(S_{0}\right)}(X+\alpha)_{\phi_{t}^{U}\left(S_{0}\right)}\right)_{f}\left(A_{\phi_{t}^{U}\left(S_{0}\right)}\right)\right)\right.
\end{aligned}
$$




$$
\begin{aligned}
& \left.-\left(\left(\widehat{d \pi}_{S_{0}}^{-1} \varphi\left(S_{0}\right) \widehat{d \pi}_{S_{0}}(X+\alpha)_{S_{0}}\right)_{f}\left(A_{S_{0}}\right)\right)\right) \\
= & \lim _{t \rightarrow 0} \frac{1}{t}\left(\left(\varphi\left(\phi_{t}^{U}\left(S_{0}\right)\right)(\bar{X}+\bar{\alpha})_{x_{0}}\right)_{f}\left(\bar{A}_{x_{0}}\right)\right) \\
& \left.-\left(\left(\varphi\left(S_{0}\right)(\bar{X}+\bar{\alpha})_{X_{0}}\right)_{f}\left(\bar{A}_{x_{0}}\right)\right)\right) \\
= & \left(\varphi\left(\lim _{t \rightarrow 0} \frac{1}{t}\left(\phi_{t}^{U}\left(S_{0}\right)-S_{0}\right)\right)(\bar{X}+\bar{\alpha})_{x_{0}}\right)_{f}\left(\bar{A}_{x_{0}}\right) \\
= & \left(\varphi\left(U_{S_{0}}\right)(\bar{X}+\bar{\alpha})_{X_{0}}\right)_{f}\left(\bar{A}_{x_{0}}\right) \\
= & \left(\varphi\left(U_{S_{0}}\right)(\bar{X}+\bar{\alpha})_{x_{0}}\right)_{f}\left(\bar{A}_{x_{0}}\right) .
\end{aligned}
$$

Thus,

$$
\widetilde{d \pi}_{S_{0}}\left(\mathcal{L}_{I U}(\mathbb{J}(X+\alpha))_{f}\right)_{S_{0}}=\left(\varphi\left(S_{0} \cdot U_{S_{0}}\right)(\bar{X}+\bar{\alpha})_{\chi_{0}}\right)_{f},
$$

and

$$
\begin{aligned}
\widetilde{d \pi}_{S_{0}}\left(\mathbb{J}\left(\mathcal{L}_{U}(\mathbb{J}(X+\alpha))_{f}\right)\right)_{S_{0}} & =\widetilde{d \pi}_{S_{0}}\left(\left(\widehat{d \pi}_{S_{0}} \varphi\left(S_{0}\right) \widehat{d \pi}_{S_{0}}\left(X_{x_{0}}+\alpha_{x_{0}}\right)\right)_{f}\right) \\
& =\left(\varphi\left(S_{0}\right) \varphi\left(U_{S_{0}}\right)(\bar{X}+\bar{\alpha})_{x_{0}}\right)_{f} \\
& =\left(\varphi\left(S_{0} \cdot U_{S_{0}}\right)(\bar{X}+\bar{\alpha})_{x_{0}}\right)_{f},
\end{aligned}
$$

where we have used that $\varphi$ is a fibre-wise algebra homomorphism.

Claim 6.

$$
\left(\mathcal{L}_{(\mathbb{J}(X+\alpha))_{v}}\left(\eta \circ I_{z}\right)\right)^{h}-\left(\mathbb{J}\left(\left(\mathcal{L}_{X}(\eta \circ I)\right)^{h}\right)\right)+\mathbb{J}\left(\left(\mathcal{L}_{(\mathbb{J}(X+\alpha))_{v}} \eta\right)^{h}\right)+\left(\mathcal{L}_{X} \eta\right)^{h}=0 .
$$

For a vertical form $\xi$

$$
\begin{aligned}
\left\langle\left(\mathcal{L}_{X} \xi\right)^{h}, Y+\beta\right\rangle & =\frac{1}{2}\left(\mathcal{L}_{X} \xi\right)^{h}(Y) \\
& =\frac{1}{2}(X(\xi(Y))-\xi([X, Y])) \\
& =-\frac{1}{2} \xi\left([X, Y]^{v}\right),
\end{aligned}
$$

and by Lemma 4.4

$$
\left\langle\mathbb{J}\left(\left(\mathcal{L}_{X} \xi\right)^{h}\right), Y+\beta\right\rangle=\frac{1}{2} \xi\left(\left[X,(\mathbb{J}(Y+\beta))_{v}\right]^{v}\right) .
$$

Thus, by Lemma 4.5,

$$
\begin{aligned}
\left\langle\left(\mathcal{L}_{(\mathbb{J}(X+\alpha))_{v}}(\eta \circ I)\right)^{h}, Y+\beta\right\rangle_{S_{0}} & =-\frac{1}{2} \eta_{S_{0}}\left(I\left[(\mathbb{J}(X+\alpha))_{v},(Y+\beta)_{v}\right]_{S_{0}}^{v}\right) \\
& =-\frac{1}{2} \eta_{S_{0}}\left(S_{0} \cdot R\left(\left(\varphi\left(S_{0}\right)\left(\bar{X}_{x_{0}}+\bar{\alpha}_{x_{0}}\right)\right)_{v},\left(\bar{Y}_{x_{0}}+\bar{\beta}_{x_{0}}\right)_{v}\right)\left(S_{0}\right)\right), \\
-\left\langle\left(\mathbb{J}\left(\left(\mathcal{L}_{X}(\eta \circ I)\right)^{h}\right)\right), Y+\beta\right\rangle_{S_{0}} & =-\frac{1}{2} \eta_{S_{0}}\left(\mathbb{J}\left[X,(\mathbb{J}(Y+\beta))_{v}\right]_{S_{0}}^{v}\right) \\
& =-\frac{1}{2} \eta_{S_{0}}\left(\mathbb{J}\left[(X+\alpha)_{v},(\mathbb{J}(Y+\beta))_{v}\right]_{S_{0}}^{v}\right) \\
& =-\frac{1}{2} \eta_{S_{0}}\left(S_{0} \cdot R\left(\left(\bar{X}_{x_{0}}+\bar{\alpha}_{x_{0}}\right)_{v},\left(\varphi\left(S_{0}\right)\left(\bar{Y}_{x_{0}}+\bar{\beta}_{x_{0}}\right)\right)_{v}\right)\left(S_{0}\right)\right), \\
\left\langle\mathbb{J}\left(\left(\mathcal{L}_{(\mathbb{J}(X+\alpha))_{v}} \eta\right)^{h}\right), Y+\beta\right\rangle_{S_{0}} & =\frac{1}{2} \eta_{S_{0}}\left(\left[(\mathbb{J}(X+\alpha))_{v},(\mathbb{J}(Y+\beta))_{v}\right]_{S_{0}}^{v}\right) \\
& =\frac{1}{2} \eta_{S_{0}}\left(R\left(\left(\varphi\left(S_{0}\right)\left(\bar{X}_{x_{0}}+\bar{\alpha}_{x_{0}}\right)\right)_{v},\left(\varphi\left(S_{0}\right)\left(\bar{Y}_{x_{0}}+\bar{\beta}_{x_{0}}\right)\right)_{v}\right)\left(S_{0}\right)\right), \\
\left\langle\left(\mathcal{L}_{X} \eta\right)^{h}, Y+\beta\right\rangle_{S_{0}} & =-\frac{1}{2} \eta_{S_{0}}\left([X, Y]_{S_{0}}^{v}\right) \\
& =-\frac{1}{2} \eta_{S_{0}}\left(R\left(\bar{X}_{x_{0}}, \bar{Y}_{x_{0}}\right)\left(S_{0}\right)\right)
\end{aligned}
$$




$$
=-\frac{1}{2} \eta_{S_{0}}\left(R\left(\left(\bar{X}_{x_{0}}+\bar{\alpha}_{x_{0}}\right)_{v},\left(\bar{Y}_{x_{0}}+\bar{\beta}_{x_{0}}\right)_{v}\right)\left(S_{0}\right)\right)
$$

Thus, if the curvature satisfies (1)

$$
\left\langle\left(\mathcal{L}_{(\mathbb{J}(X+\alpha))_{v}}(\eta \circ I)\right)^{h}-\left(\mathbb{J}\left(\left(\mathcal{L}_{X}(\eta \circ I)\right)^{h}\right)\right)+\mathbb{J}\left(\left(\mathcal{L}_{(\mathbb{J}(X+\alpha))_{v}} \eta\right)^{h}\right)+\left(\mathcal{L}_{X} \eta\right)^{h}, Y+\beta\right\rangle=0 .
$$

\subsection{Proof of Lemma 5.3}

Let $X, Y$ be basic vector fields, i.e. horizontal lifts of vectors $\bar{X}, \bar{Y}$ on $M$, and $\alpha, \beta$ be pullbacks of 1-forms $\bar{\alpha}, \bar{\beta}$ on $M$. We will prove the following two lemmas.

Lemma 5.4. If the generalized torsion $\mathcal{T}$ satisfies condition (2), then

$$
\left(\mathcal{N}^{\mathbb{J}}(X+\alpha, Y+\beta)\right)^{h}=0 .
$$

Lemma 5.5. If the curvature $R$ satisfies condition (1), then

$$
\left(\mathcal{N}^{\mathbb{J}}(X+\alpha, Y+\beta)\right)^{v}=0 .
$$

\subsubsection{Proof of Lemma 5.4}

In order to carry out the calculations, let us consider

- $S_{0} \in Z$ and $x_{0}=\pi\left(S_{0}\right) \in M$;

- $\bar{A}_{x_{0}} \in T_{x_{0}} M$ and $A_{S_{0}} \in H_{S_{0}}$ the corresponding horizontal vector (whenever necessary, we will consider a local extension $\bar{A}$ of $\bar{A}_{x_{0}}$ and the corresponding local basic field $A$ );

- $S(x)$ a local section of $Z$ on an open set $B \subset M$ around $x_{0}$ such that it is tangent to $H_{S_{0}}$ and $(\nabla \bar{S})_{x_{0}}=0$. Such a section defines a local almost complex structure by

$$
\bar{S}_{x}:=\varphi(S(x)): \mathbb{T}_{x} M \longrightarrow \mathbb{T}_{x} M, \quad \bar{S}^{2}=-\mathrm{Id}_{\mathbb{T}_{x} M} ;
$$

- a curve $\gamma(t)$ in the image of $\bar{S}(x)$ such that $\gamma(0)=S_{0}$ and $\dot{\gamma}(0)=Y_{S_{0}}$ (it exists due to the tangency to $H_{S_{0}}$ ). Clearly, the curve $\bar{\gamma}(t)=\pi(\gamma(t))$ in $M$ is such that $\bar{\gamma}(0)=x_{0}, \dot{\gamma}(0)=\bar{Y}_{x_{0}}$ and $\gamma(t)=\bar{S}(\bar{\gamma}(t))$;

- a curve $\delta(t)$ in the image of $\bar{S}(x)$ such that $\delta(0)=S_{0}$ and $\dot{\gamma}(0)=A_{S_{0}}$ (it exists due to the tangency to $H_{S_{0}}$ ). Clearly, the curve $\bar{\delta}(t)=\pi(\delta(t))$ in $M$ is such that $\bar{\delta}(0)=x_{0}, \dot{\delta}(0)=\bar{A}_{x_{0}}$ and $\delta(t)=\bar{S}(\bar{\delta}(t))$;

- $f: M \rightarrow \mathbb{R}$ a smooth function.

We will actually prove the following.

Lemma 5.6. Let $S_{0} \in Z$ and $x_{0}=\pi\left(S_{0}\right) \in M$. Then

$$
\widehat{d \pi}_{S_{0}} \mathcal{N}^{\mathbb{J}}\left(X_{S_{0}}+\alpha_{S_{0}}, Y_{S_{0}}+\beta_{S_{0}}\right)=\mathcal{N}^{\bar{S}}\left(\bar{X}_{x_{0}}+\bar{\alpha}_{x_{0}}, \bar{Y}_{x_{0}}+\bar{\beta}_{\chi_{0}}\right) .
$$

Proof. Recall that

$$
\mathcal{N}^{\rrbracket}(X+\alpha, Y+\beta)=\llbracket \mathbb{J}(X+\alpha), \mathbb{J}(Y+\beta) \rrbracket-\rrbracket \llbracket \rrbracket(X+\alpha), Y+\beta \rrbracket-\mathbb{\rrbracket} \llbracket X+\alpha, \mathbb{J}(Y+\beta) \rrbracket-\llbracket X+\alpha, Y+\beta \rrbracket .
$$

We will examine one term at a time.

\section{Lemma 5.7.}

$$
\widehat{d \pi}_{S_{0}} \llbracket X+\alpha, Y+\beta \rrbracket_{S}=\llbracket \bar{X}+\bar{\alpha}, \bar{Y}+\bar{\beta} \rrbracket_{x_{0}} .
$$


Proof. We have

$$
\begin{aligned}
\widehat{d \pi} \llbracket X+\alpha, Y+\beta \rrbracket & =\widehat{d \pi}\left([X, Y]+\mathcal{L}_{X} \beta-\mathcal{L}_{Y} \alpha-\frac{1}{2} d(\beta(X)-\alpha(Y))\right) \\
& =d \pi([X, Y])+\widetilde{d \pi}\left(\mathcal{L}_{X} \beta\right)-\widetilde{d \pi}\left(\mathcal{L}_{Y} \alpha\right)-\frac{1}{2} \widetilde{d \pi}(d(\beta(X)-\alpha(Y))) \\
& =[\bar{X}, \bar{Y}]+\widetilde{d \pi}\left(\mathcal{L}_{X} \beta\right)-\widetilde{d \pi}\left(\mathcal{L}_{Y} \alpha\right)-\frac{1}{2} \widetilde{d \pi}(d(\beta(X)-\alpha(Y))) .
\end{aligned}
$$

Claim 1.

$$
\left(\widetilde{d \pi}\left(d^{Z}(\beta(X))\right)_{S_{0}}=d^{M}(\bar{\beta}(\bar{X}))_{x_{0}} .\right.
$$

Indeed,

$$
\begin{aligned}
\left(\widetilde{d \pi}\left(d^{Z}(\beta(X))\right)_{S_{0}}\left(\bar{A}_{x_{0}}\right)\right. & =\widetilde{d \pi}_{S_{0}}\left(d^{Z}(\beta(X))_{S_{0}}\left(\bar{A}_{x_{0}}\right)\right. \\
& =\left(d^{Z}\left(\pi^{\star} \bar{\beta}(X)\right)_{S_{0}}\left(A_{S_{0}}\right)\right. \\
& =\left(d^{M}(\bar{\beta}(d \pi X) \circ \pi)_{S_{0}}\left(A_{S_{0}}\right)\right. \\
& =\left(d^{M}(\bar{\beta}(\bar{X}))_{x_{0}} \circ d \pi_{S_{0}}\right)\left(A_{S_{0}}\right) \\
& =d^{M}(\bar{\beta}(\bar{X}))_{X_{0}}\left(d \pi_{S_{0}}\left(A_{S_{0}}\right)\right) \\
& =d^{M}(\bar{\beta}(\bar{X}))_{X_{0}}\left(\bar{A}_{x_{0}}\right) .
\end{aligned}
$$

Claim 2.

$$
\widetilde{d \pi}_{S_{0}}\left(\mathcal{L}_{X} \beta\right)_{S_{0}}=\left(\mathcal{L}_{\bar{X}} \bar{\beta}\right)_{\chi_{0}} .
$$

Since

$$
\left.\mathcal{L}_{X} \beta=d(\beta(X))+X\right\lrcorner d \beta
$$

we have

$$
\begin{aligned}
\left(\widetilde{d \pi}_{S_{0}}\left(\mathcal{L}_{X} \beta\right)_{S_{0}}\right)\left(\bar{A}_{x_{0}}\right) & =\left(\widetilde{d \pi}_{S_{0}}\left(d^{Z}(\beta(X)+X\lrcorner d^{Z} \beta\right)_{S}\right)\left(\bar{A}_{X_{0}}\right) \\
& =\left(\widetilde{d \pi}_{S_{0}}\left(d^{Z}(\beta(X))_{S_{0}}+\widetilde{d \pi}_{S_{0}}\left((X\lrcorner d^{Z} \beta\right)_{S_{0}}\right)\right)\left(\bar{A}_{X_{0}}\right) \\
& \left.=d^{M}(\bar{\beta}(\bar{X}))_{X_{0}}\left(\bar{A}_{X_{0}}\right)+\widetilde{d \pi}_{S_{0}}\left((X\lrcorner d^{Z} \beta\right)_{S_{0}}\right)\left(\bar{A}_{X_{0}}\right) .
\end{aligned}
$$

Now,

$$
\begin{aligned}
\left.\widetilde{d \pi}_{S_{0}}\left((X\lrcorner d^{Z} \beta\right)_{S_{0}}\right)\left(\bar{A}_{\chi_{0}}\right) & \left.=\widetilde{d \pi}_{S_{0}}\left((X\lrcorner d^{Z}\left(\pi^{\star} \bar{\beta}\right)\right)_{S_{0}}\right)\left(\bar{A}_{\chi_{0}}\right) \\
& \left.=\left((X\lrcorner \pi^{\star} d^{M}(\bar{\beta})\right)_{S_{0}}\right)\left(A_{S_{0}}\right) \\
& =d^{M} \bar{\beta}\left(d \pi_{S_{0}} X_{S_{0}}, d \pi_{S_{0}}\left(A_{S_{0}}\right)\right) \\
& =d^{M} \bar{\beta}\left(\bar{X}, \bar{A}_{x_{0}}\right) \\
& \left.=(\bar{X}\lrcorner d^{M} \bar{\beta}\right)_{x_{0}}\left(\bar{A}_{x_{0}}\right) .
\end{aligned}
$$

which concludes the proof of Claim 2 and Lemma 5.7.

Lemma 5.8 .

$$
\begin{aligned}
& \widehat{d \pi}_{S_{0}} \mathbb{\amalg} \llbracket \mathbb{J}(X+\alpha), Y+\beta \rrbracket S_{S_{0}}=\quad \bar{S}_{X_{0}} \llbracket \bar{S}(\bar{X}+\bar{\alpha}), \bar{Y}+\bar{\beta} \rrbracket x_{x_{0}}, \\
& \widehat{d \pi}_{S_{0}} \mathbb{J} \llbracket X+\alpha, \mathbb{J}(Y+\beta) \rrbracket_{S_{0}}=\quad \bar{S}_{X_{0}} \llbracket \bar{X}+\bar{\alpha}, \bar{S}(\bar{Y}+\bar{\beta}) \rrbracket_{x_{0}} \text {. }
\end{aligned}
$$


Proof. We will only prove the first identity since the second one is analogous. Consider

$$
\begin{aligned}
& \widehat{d \pi}(\rrbracket \llbracket \rrbracket(X+\alpha), Y+\beta \rrbracket)=\widehat{d \pi}(\mathbb{\rrbracket} \llbracket \rrbracket(X+\alpha), Y+\beta \rrbracket)^{h} \\
& =\widehat{d \pi} \mathbb{J}(\llbracket \mathbb{J}(X+\alpha), Y+\beta \rrbracket)^{h} \\
& =\widehat{d \pi} \widehat{d \pi}^{-1} \varphi(S) \widehat{d \pi} \llbracket \rrbracket(X+\alpha), Y+\beta \rrbracket^{h} \\
& =\varphi(S) \widehat{d \pi} \llbracket \rrbracket(X+\alpha), Y+\beta \rrbracket \\
& =\bar{S} \widehat{d \pi} \llbracket \rrbracket(X+\alpha), Y+\beta \rrbracket \text {. }
\end{aligned}
$$

Now

$$
\begin{aligned}
& \widehat{d \pi} \llbracket \mathbb{J}(X+\alpha), Y+\beta \rrbracket=\widehat{d \pi}\left(\left[(\mathbb{J}(X+\alpha))_{v}, Y\right]+\mathcal{L}_{(\mathbb{J}(X+\alpha))_{v}} \beta-\mathcal{L}_{Y}(\mathbb{J}(X+\alpha))_{f}\right. \\
& \left.-\frac{1}{2} d\left(\beta\left((\mathbb{J}(X+\alpha))_{v}\right)-(\mathbb{J}(X+\alpha))_{f}(Y)\right)\right) \\
& =d \pi\left[(\mathbb{J}(X+\alpha))_{v}, Y\right]+\widetilde{d \pi} \mathcal{L}_{(\mathbb{J}(X+\alpha))_{v}} \beta-\widetilde{d \pi} \mathcal{L}_{Y}(\mathbb{J}(X+\alpha))_{f} \\
& -\frac{1}{2} \widetilde{d \pi}\left(d\left(\beta\left((\mathbb{J}(X+\alpha))_{v}\right)-(\mathbb{J}(X+\alpha))_{f}(Y)\right)\right) \text {. }
\end{aligned}
$$

Claim 1.

$$
d \pi_{S_{0}}\left[(\mathbb{J}(X+\alpha))_{v}, Y\right]_{S_{0}}=\left[(\bar{S}(\bar{X}+\bar{\alpha}))_{v}, \bar{Y}\right]_{x_{0}}
$$

Indeed, let $f: M \rightarrow \mathbb{R}$ and consider

$$
\begin{aligned}
d \pi_{S_{0}}\left[(\mathbb{J}(X+\alpha))_{v}, Y\right]_{S_{0}}(f) & =d f_{x_{0}}^{M} d \pi_{S_{0}}\left[(\mathbb{J}(X+\alpha))_{v}, Y\right]_{S_{0}} \\
& =d^{Z}(f \circ \pi)_{S_{0}}\left[(\mathbb{J}(X+\alpha))_{v}, Y\right]_{S_{0}} \\
& \left.\left.=\left((\mathbb{J}(X+\alpha))_{V}\right)\right)_{S_{0}}(Y(f \circ \pi))-Y_{S_{0}}\left((\mathbb{J}(X+\alpha))_{V}\right)(f \circ \pi)\right) .
\end{aligned}
$$

The term

$$
\begin{aligned}
\left((\mathbb{d}(X+\alpha))_{v}\right)_{S_{0}}(Y(f \circ \pi)) & =d^{Z}(Y(f \circ \pi))_{S_{0}}\left(\left((\mathbb{J}(X+\alpha))_{v}\right)_{S_{0}}\right) \\
& =d^{Z}\left(d^{M} f \circ d \pi(Y)\right)_{S_{0}}\left(\left(\widehat{d \pi}_{S_{0}}^{-1} \varphi\left(S_{0}\right) \widehat{d \pi}_{S_{0}}\left(X_{S_{0}}+\alpha_{S_{0}}\right)\right)_{V}\right) \\
& =d^{Z}\left(d^{M} f(\bar{Y}) \circ \pi\right)_{S_{0}}\left(\left(\widehat{d \pi}_{S_{0}}^{-1} \varphi\left(S_{0}\right)\left(\bar{X}_{x_{0}}+\bar{\alpha}_{x_{0}}\right)\right)_{v}\right) \\
& =d^{Z}(\bar{Y}(f) \circ \pi)_{S_{0}}\left(\left(\widehat{d \pi}_{S_{0}}^{-1} \varphi\left(S_{0}\right)\left(\bar{X}_{x_{0}}+\bar{\alpha}_{x_{0}}\right)\right)_{v}\right) \\
& =d^{M}(\bar{Y}(f))_{x_{0}} d \pi_{S_{0}}\left(\left(\widehat{d \pi}_{S_{0}}^{-1} \varphi\left(S_{0}\right)\left(\bar{X}_{x_{0}}+\bar{\alpha}_{x_{0}}\right)\right)_{V}\right) \\
& =d^{M}(\bar{Y}(f))_{x_{0}}\left(\left(\widehat{d \pi}_{S_{0}} \widehat{d \pi}_{S_{0}}^{-1} \varphi\left(S_{0}\right)\left(\bar{X}_{x_{0}}+\bar{\alpha}_{x_{0}}\right)\right)_{V}\right) \\
& =d^{M}(\bar{Y}(f))_{x_{0}}\left(\left(\varphi\left(S_{0}\right)\left(\bar{X}_{x_{0}}+\bar{\alpha}_{x_{0}}\right)\right)_{v}\right) .
\end{aligned}
$$

The other term is

$$
\begin{aligned}
& Y_{S_{0}}\left((\mathbb{J}(X+\alpha))_{v}(f \circ \pi)\right)=d^{Z}\left((\mathbb{\mathbb { J }}(X+\alpha))_{v}(f \circ \pi)\right)_{S_{0}}\left(Y_{S_{0}}\right) \\
& =\lim _{t \rightarrow 0} \frac{1}{t}\left(\left((\mathbb{J}(X+\alpha))_{v}(f \circ \pi)\right)(\gamma(t))-\left((\mathbb{J}(X+\alpha))_{v}(f \circ \pi)\right)\left(S_{0}\right)\right) \\
& =\lim _{t \rightarrow 0} \frac{1}{t}\left(d^{M} f_{\pi(\gamma(t))} d \pi_{\gamma(t)}\left((\mathbb{J}(X+\alpha))_{v}\right)_{\gamma(t)}-d^{M} f_{\pi\left(S_{0}\right)} d \pi_{S_{0}}\left((\mathbb{J}(X+\alpha))_{v}\right)_{S_{0}}\right) \\
& =\lim _{t \rightarrow 0} \frac{1}{t}\left(d^{M} f_{\bar{\gamma}(t)} d \pi_{\gamma(t)}\left(\widehat{d \pi}_{\gamma(t)}^{-1} \varphi(\gamma(t)) \widehat{d \pi}_{\gamma(t)}\left(X_{\gamma(t)}+\alpha_{\gamma(t)}\right)\right)_{v}-d^{M} f_{X_{0}} d \pi_{S_{0}}\left(\widehat{d \pi}_{S_{0}}^{-1} \varphi\left(S_{0}\right) \widehat{d \pi}_{S_{0}}\left(X_{S_{0}}+\alpha_{S_{0}}\right)\right)_{V}\right) \\
& =\lim _{t \rightarrow 0} \frac{1}{t}\left(d^{M} f_{\bar{\gamma}(t)}\left(\widehat{d \pi}_{\gamma(t)} \widehat{d \pi}_{\gamma(t)}^{-1} \varphi(\gamma(t))\left(\bar{X}_{\gamma(t)}+\bar{\alpha}_{\gamma(t)}\right)\right)_{v}-d^{M} f_{x_{0}}\left(\widehat{d \pi}_{S_{0}} \widehat{d \pi}_{S_{0}}^{-1} \varphi\left(S_{0}\right)\left(\bar{X}_{x_{0}}+\bar{\alpha}_{x_{0}}\right)\right)_{v}\right) \\
& =\lim _{t \rightarrow 0} \frac{1}{t}\left(d^{M} f_{\bar{\gamma}(t)}\left(\varphi(S(\bar{\gamma}(t)))\left(\bar{X}_{\bar{\gamma}(t)}+\bar{\alpha}_{\bar{\gamma}(t)}\right)\right)_{v}-d^{M} f_{x_{0}}\left(\varphi\left(S\left(x_{0}\right)\right)\left(\bar{X}_{x_{0}}+\bar{\alpha}_{x_{0}}\right)\right)_{v}\right) \\
& =\lim _{t \rightarrow 0} \frac{1}{t}\left(d^{M} f_{\bar{\gamma}(t)}\left((\bar{S}(\bar{X}+\bar{\alpha}))_{v}\right)_{\bar{\gamma}(t)}-d^{M} f_{x_{0}}\left((\bar{S}(\bar{X}+\bar{\alpha}))_{v}\right)_{x_{0}}\right) \\
& =\lim _{t \rightarrow 0} \frac{1}{t}\left(\left((\bar{S}(\bar{X}+\bar{\alpha}))_{v}(f)\right)(\bar{\gamma}(t))-\left((\bar{S}(\bar{X}+\bar{\alpha}))_{v}(f)\right)\left(x_{0}\right)\right) \\
& =\bar{Y}_{x_{0}}\left((\bar{S}(\bar{X}+\bar{\alpha}))_{v}(f)\right)
\end{aligned}
$$


Note that this calculation only required the value $Y_{S_{0}}$ to be horizontal.

Claim 2.

$$
\widetilde{d \pi}_{S_{0}} d^{Z}\left(\beta\left((\mathbb{J}(X+\alpha))_{v}\right)\right)_{S_{0}}=d^{M}\left(\bar{\beta}\left((\bar{S}(\bar{X}+\bar{\alpha}))_{V}\right)\right)_{x_{0}}
$$

Indeed,

$$
\begin{aligned}
d^{Z}\left(\beta\left((\mathbb{J}(X+\alpha))_{v}\right)\right)_{S_{0}}\left(A_{S_{0}}\right) & =\lim _{t \rightarrow 0} \frac{1}{t}\left(\left(\beta\left((\mathbb{J}(X+\alpha))_{v}\right)\right)(\delta(t))-\left(\beta\left((\mathbb{J}(X+\alpha))_{v}\right)\right)\left(S_{0}\right)\right) \\
& =\lim _{t \rightarrow 0} \frac{1}{t}\left(\beta_{\delta(t)}\left(\left(\mathbb{J}(X+\alpha)_{\delta(t)}\right)_{v}\right)-\beta_{S_{0}}\left(\left(\mathbb{J}(X+\alpha)_{S_{0}}\right)_{v}\right)\right) \\
& =\lim _{t \rightarrow 0} \frac{1}{t}\left(\left(\pi^{\star} \bar{\beta}\right)_{\delta(t)}\left(\left(\widehat{d \pi}_{\delta(t)}^{-1} \varphi(\delta(t)) \widehat{d \pi}_{\delta(t)}\left(X_{\delta(t)}+\alpha_{\delta(t)}\right)\right)_{v}\right)-\left(\pi^{\star} \bar{\beta}\right)_{S_{0}}\left(\left(\widehat{d \pi}_{S_{0}}^{-1} \varphi\left(S_{0}\right) \widehat{d \pi}_{S_{0}}\left(X_{S_{0}}+\alpha_{S_{0}}\right)\right)_{v}\right)\right) \\
& =\lim _{t \rightarrow 0} \frac{1}{t}\left(\bar{\beta}_{\bar{\delta}(t)} d \pi_{\delta(t)}\left(\left(\widehat{d \pi}_{\delta(t)}^{-1} \varphi(S(\bar{\delta}(t)))\left(\bar{X}_{\bar{\delta}(t)}+\bar{\alpha}_{\bar{\delta}(t)}\right)\right)_{v}\right)-\bar{\beta}_{x_{0}} d \pi_{S_{0}}\left(\left(\widehat{d \pi}_{S_{0}}^{-1} \varphi\left(S\left(x_{0}\right)\right)\left(\bar{X}_{x_{0}}+\bar{\alpha}_{x_{0}}\right)\right)_{v}\right)\right) \\
& =\lim _{t \rightarrow 0} \frac{1}{t}\left(\bar{\beta}_{\bar{\delta}(t)}\left(\left(\widehat{d \pi}_{\delta(t)} \widehat{d \pi}_{\delta(t)}^{-1} \varphi(S(\bar{\delta}(t)))\left(\bar{X}_{\bar{\delta}(t)}+\bar{\alpha}_{\bar{\delta}(t)}\right)\right)_{v}\right)-\bar{\beta}_{x_{0}}\left(\left(\widehat{d \pi}_{S_{0}} \widehat{d \pi}_{S_{0}}^{-1} \varphi\left(S\left(x_{0}\right)\right)\left(\bar{X}_{x_{0}}+\bar{\alpha}_{x_{0}}\right)\right)_{v}\right)\right) \\
& =\lim _{t \rightarrow 0} \frac{1}{t}\left(\bar{\beta}_{\bar{\delta}(t)}\left(\left(\bar{S}_{\bar{\delta}(t)}\left(\bar{X}_{\bar{\delta}(t)}+\bar{\alpha}_{\bar{\delta}(t)}\right)\right)_{v}\right)-\bar{\beta}_{x_{0}}\left(\left(\bar{S}_{x_{0}}\left(\bar{X}_{x_{0}}+\bar{\alpha}_{x_{0}}\right)\right)_{v}\right)\right) \\
& =\lim _{t \rightarrow 0} \frac{1}{t}\left(\left(\bar{\beta}\left((\bar{S}(\bar{X}+\bar{\alpha}))_{v}\right)\right)(\bar{\delta}(t))-\left(\bar{\beta}\left((\bar{S}(\bar{X}+\bar{\alpha}))_{v}\right)\right)\left(x_{0}\right)\right) \\
& =d^{M}\left(\bar{\beta}\left((\bar{S}(\bar{X}+\bar{\alpha}))_{v}\right)\right)_{x_{0}}\left(\bar{A}_{x_{0}}\right) .
\end{aligned}
$$

Claim 3.

$$
\widetilde{d \pi}_{S_{0}}\left(\mathcal{L}_{(\mathbb{J}(X+\alpha))_{v}} \beta\right)_{S_{0}}=\left(\mathcal{L}_{(\bar{S}(\bar{X}+\bar{\alpha}))_{v}} \bar{\beta}\right)_{X_{0}}
$$

Indeed,

$$
\begin{aligned}
\widetilde{d \pi}_{S_{0}}\left(\mathcal{L}_{(\mathbb{J}(X+\alpha))_{v}} \beta\right)_{S_{0}}\left(\bar{A}_{\chi_{0}}\right) & =\left(\mathcal{L}_{(\mathbb{J}(X+\alpha))_{v}} \beta\right)_{S_{0}}\left(A_{S_{0}}\right) \\
& =\left(d^{Z}\left(\beta\left((\mathbb{J}(X+\alpha))_{V}\right)\right)_{S_{0}}\left(A_{S_{0}}\right)+\left(d^{Z} \beta\right)_{S_{0}}\left(\left((\mathbb{J}(X+\alpha))_{v}\right)_{S_{0}}, A_{S_{0}}\right)\right.
\end{aligned}
$$

Now

$$
\begin{aligned}
\left(d^{Z} \beta\right)_{S_{0}}\left(\left((\mathbb{J}(X+\alpha))_{v}\right)_{S_{0}}, A_{S_{0}}\right) & =\left(d^{Z} \pi^{\star} \bar{\beta}\right)_{S_{0}}\left(\left(\widehat{d \pi}_{S_{0}}^{-1} \varphi\left(S_{0}\right) \widehat{d \pi}_{S_{0}}\left(X_{S_{0}}+\alpha_{S_{0}}\right)\right)_{v}, A_{S_{0}}\right) \\
& =\left(\pi^{\star} d^{M} \bar{\beta}\right)_{S_{0}}\left(\left(\widehat{d \pi}_{S_{0}}^{-1} \varphi\left(S_{0}\right)\left(\bar{X}_{x_{0}}+\bar{\alpha}_{x_{0}}\right)\right)_{v}, A_{S_{0}}\right) \\
& =\left(d^{M} \bar{\beta}\right)_{x_{0}}\left(d \pi_{S_{0}}\left(\widehat{d \pi}_{S_{0}}^{-1} \varphi\left(S_{0}\right)\left(\bar{X}_{x_{0}}+\bar{\alpha}_{x_{0}}\right)\right)_{v}, d \pi_{S_{0}} A_{S_{0}}\right) \\
& =\left(d^{M} \beta\right)_{x_{0}}\left(\left(\widehat{d \pi}_{S_{0}} \widehat{d \pi}_{S_{0}}^{-1} \varphi\left(S_{0}\right)\left(\bar{X}_{x_{0}}+\bar{\alpha}_{x_{0}}\right)\right)_{v}, \bar{A}_{x_{0}}\right) \\
& =\left(d^{M} \beta\right)_{x_{0}}\left(\left(\bar{S}_{x_{0}}\left(\bar{X}_{x_{0}}+\bar{\alpha}_{x_{0}}\right)\right)_{v}, \bar{A}_{x_{0}}\right) .
\end{aligned}
$$

Claim 4

$$
\widetilde{d \pi}_{S_{0}} d^{Z}\left((\mathbb{J}(X+\alpha))_{f}(Y)\right)_{S_{0}}=d^{M}\left((\bar{S}(\bar{X}+\bar{\alpha}))_{f}(\bar{Y})\right)_{X_{0}}
$$

Indeed,

$$
\begin{aligned}
\left(\widehat{d \pi}_{S_{0}} d^{Z}\left((\mathbb{J}(X+\alpha))_{f}(Y)\right)_{S_{0}}\right)\left(\bar{A}_{x_{0}}\right) & =\left(d^{Z}\left((\mathbb{J}(X+\alpha))_{f}(Y)\right)\right)_{S_{0}}\left(A_{S_{0}}\right) \\
& =\lim _{t \rightarrow 0} \frac{1}{t}\left(\left((\mathbb{J}(X+\alpha))_{f}(Y)\right)(\delta(t))-\left((\mathbb{J}(X+\alpha))_{f}(Y)\right)\left(S_{0}\right)\right) \\
& =\lim _{t \rightarrow 0} \frac{1}{t}\left(\left(\widehat{d \pi}_{\delta(t)}^{-1} \varphi(\delta(t)) \widehat{d \pi}_{\delta(t)}\left(X_{\delta(t)}+\alpha_{\delta(t)}\right)\right)_{f}\left(Y_{\delta(t)}\right)-\left(\widehat{d \pi}_{S_{0}}^{-1} \varphi\left(S_{0}\right) \widehat{d \pi}_{S_{0}}\left(X_{S_{0}}+\alpha_{S_{0}}\right)\right)_{f}\left(Y_{S_{0}}\right)\right) \\
& =\lim _{t \rightarrow 0} \frac{1}{t}\left(\left(\widehat{d \pi}_{\delta(t)}^{-1} \varphi(S(\bar{\delta}(t)))\left(\bar{X}_{\bar{\delta}(t)}+\bar{\alpha}_{\bar{\delta}(t)}\right)\right)_{f}\left(Y_{\delta(t)}\right)-\left(\widehat{d \pi}_{S_{0}}^{-1} \varphi\left(S_{0}\right)\left(\bar{X}_{x_{0}}+\bar{\alpha}_{x_{0}}\right)\right)_{f}\left(Y_{S_{0}}\right)\right) \\
& =\lim _{t \rightarrow 0} \frac{1}{t}\left(\left(\varphi(S(\bar{\delta}(t)))\left(\bar{X}_{\bar{\delta}(t)}+\bar{\alpha}_{\bar{\delta}(t)}\right)\right)_{f}\left(d \pi_{\delta(t)} Y_{\delta(t)}\right)-\left(\varphi\left(S_{0}\right)\left(\bar{X}_{x_{0}}+\bar{\alpha}_{x_{0}}\right)\right)_{f}\left(d \pi_{S_{0}} Y_{S_{0}}\right)\right)
\end{aligned}
$$




$$
\begin{aligned}
& =\lim _{t \rightarrow 0} \frac{1}{t}\left(\left(\bar{S}_{\bar{\delta}(t)}\left(\bar{X}_{\bar{\delta}(t)}+\bar{\alpha}_{\bar{\delta}(t)}\right)\right)_{f}\left(\bar{Y}_{\bar{\delta}(t)}\right)-\left(\bar{S}_{x_{0}}\left(\bar{X}_{x_{0}}+\bar{\alpha}_{x_{0}}\right)\right)_{f}\left(\bar{Y}_{x_{0}}\right)\right) \\
& =\lim _{t \rightarrow 0} \frac{1}{t}\left(\left((\bar{S}(\bar{X}+\bar{\alpha}))_{f}(\bar{Y})\right)(\bar{\delta}(t))-\left((\bar{S}(\bar{X}+\bar{\alpha}))_{f}(\bar{Y})\right)\left(x_{0}\right)\right) \\
& =d^{M}\left((\bar{S}(\bar{X}+\bar{\alpha}))_{f}(\bar{Y})\right)_{x_{0}}\left(\bar{A}_{x_{0}}\right) \text {. }
\end{aligned}
$$

\section{$\underline{\text { Claim } 5}$}

$$
\widetilde{d \pi}_{S_{0}}\left(\mathcal{L}_{Y}(\mathbb{J}(X+\alpha))_{f}\right)_{S_{0}}=\left(\mathcal{L}_{\bar{Y}}(\bar{S}(\bar{X}+\bar{\alpha}))_{f}\right)_{x_{0}}
$$

Indeed,

$$
\begin{aligned}
\left(\widetilde{d \pi}_{S_{0}}\left(\mathcal{L}_{Y}(\mathbb{J}(X+\alpha))_{f}\right)_{S_{0}}\right)\left(\bar{A}_{x_{0}}\right) & =\left(\mathcal{L}_{Y}(\mathbb{J}(X+\alpha))_{f}\right)_{S_{0}}\left(A_{S_{0}}\right) \\
& =d^{Z}\left(\left((\mathbb{J}(X+\alpha))_{f}\right)(Y)\right)_{S_{0}}\left(A_{S_{0}}\right)+\left(d^{Z}\left((\mathbb{J}(X+\alpha))_{f}\right)\right)\left(Y_{S_{0}}, A_{S_{0}}\right) \\
& =d^{M}\left((\bar{S}(\bar{X}+\bar{\alpha}))_{f}(\bar{Y})\right)_{\chi_{0}}\left(\bar{A}_{x_{0}}\right)+\left(d^{Z}\left((\mathbb{J}(X+\alpha))_{f}\right)\right)\left(Y_{S_{0}}, A_{S_{0}}\right) .
\end{aligned}
$$

Now

$$
\begin{aligned}
\left(d^{Z}\left((\mathbb{J}(X+\alpha))_{f}\right)\left(Y_{S_{0}}, A_{S_{0}}\right)=\right. & Y_{S_{0}}\left((\mathbb{J}(X+\alpha))_{f}(A)\right)-A_{S_{0}}\left((\mathbb{J}(X+\alpha))_{f}(Y)\right)-\left((\mathbb{J}(X+\alpha))_{f}\right)_{S_{0}}\left([Y, A]_{S_{0}}\right) \\
= & d^{Z}\left((\mathbb{J}(X+\alpha))_{f}(A)\right)_{S_{0}}\left(Y_{S_{0}}\right)-d^{Z}\left((\mathbb{J}(X+\alpha))_{f}(Y)\right)_{S_{0}}\left(A_{S_{0}}\right)-\left((\mathbb{J}(X+\alpha))_{f}\right)_{S_{0}}\left([Y, A]_{S_{0}}\right) \\
= & d^{M}\left((\bar{S}(\bar{X}+\bar{\alpha}))_{f}(\bar{A})\right)_{x_{0}}\left(\bar{Y}_{x_{0}}\right)-d^{M}\left((\bar{S}(\bar{X}+\bar{\alpha}))_{f}(\bar{Y})\right)_{x_{0}}\left(\bar{A}_{x_{0}}\right) \\
& -\left(\left(\bar{d}_{S_{0}}^{-1} \varphi\left(S_{0}\right) \widehat{d \pi}_{S_{0}}\left(X_{S_{0}}+\alpha_{S_{0}}\right)\right)_{f}\right)\left([Y, A]_{S_{0}}\right) \\
= & \bar{Y}_{x_{0}}\left((\bar{S}(\bar{X}+\bar{\alpha}))_{f}(\bar{A})\right)-\bar{A}_{x_{0}}\left((\bar{S}(\bar{X}+\bar{\alpha}))_{f}(\bar{Y})\right) \\
& -\left(\bar{S}_{x_{0}}\left(\bar{X}_{x_{0}}+\bar{\alpha}_{x_{0}}\right)\right)_{f}\left(d \pi_{S_{0}}[Y, A]_{S_{0}}\right) \\
= & \bar{Y}_{x_{0}}\left((\bar{S}(\bar{X}+\bar{\alpha}))_{f}(\bar{A})\right)-\bar{A}_{x_{0}}\left((\bar{S}(\bar{X}+\bar{\alpha}))_{f}(\bar{Y})\right) \\
& -\left(\bar{S}_{x_{0}}\left(\bar{X}_{x_{0}}+\bar{\alpha}_{x_{0}}\right)\right)_{f}\left([\bar{Y}, \bar{A}]_{x_{0}}\right) \\
= & \left(d^{M}\left((\bar{S}(\bar{X}+\bar{\alpha}))_{f}\right)_{x_{0}}\left(\bar{Y}_{x_{0}}, \bar{A}_{x_{0}}\right)\right.
\end{aligned}
$$

which proves Claim 5 and Lemma 5.8.

\section{Lemma 5.9.}

$$
\widetilde{d \pi}_{S_{0}} \llbracket \mathbb{J}(X+\alpha), \mathbb{J}(Y+\beta) \rrbracket_{S_{0}}=\llbracket \bar{S}(\bar{X}+\bar{\alpha}), \bar{S}(\bar{Y}+\bar{\beta}) \rrbracket_{x_{0}} .
$$

Proof. We have

$$
\begin{aligned}
\widetilde{d \pi}_{S_{0}} \llbracket \mathbb{J}(X+\alpha), \mathbb{J}(Y+\beta) \rrbracket_{S_{0}}= & \widetilde{d \pi}_{S_{0}}\left(\left[(\mathbb{J}(X+\alpha))_{v},(\mathbb{J}(Y+\beta))_{v}\right]+\mathcal{L}_{(\mathbb{J}(X+\alpha))_{v}}(\mathbb{J}(Y+\beta))_{f}-L_{(\mathbb{J}(Y+\beta))_{v}}(\mathbb{J}(X+\alpha))_{f}\right. \\
& \left.-\frac{1}{2} d\left((\mathbb{J}(Y+\beta))_{f}\left((\mathbb{J}(X+\alpha))_{v}\right)-(\mathbb{J}(X+\alpha))_{f}\left((\mathbb{J}(Y+\beta))_{v}\right)\right)\right) \\
= & d \pi\left[(\mathbb{J}(X+\alpha))_{v},(\mathbb{J}(Y+\beta))_{v}\right]+\widetilde{d \pi} \mathcal{L}_{(\mathbb{J}(X+\alpha))_{v}}(\mathbb{J}(Y+\beta))_{f}-\widetilde{d \pi} L_{(\mathbb{J}(Y+\beta))_{v}}(\mathbb{J}(X+\alpha))_{f} \\
& -\frac{1}{2} \widetilde{d \pi} d\left((\mathbb{J}(Y+\beta))_{f}\left((\mathbb{J}(X+\alpha))_{v}\right)-(\mathbb{J}(X+\alpha))_{f}\left((\mathbb{J}(Y+\beta))_{v}\right)\right) .
\end{aligned}
$$

Claim 1.

$$
d \pi_{S_{0}}\left[(\mathbb{J}(X+\alpha))_{v},(\mathbb{J}(Y+\beta))_{V}\right]_{S_{0}}=\left[(\bar{S}(\bar{X}+\bar{\alpha}))_{v},(\bar{S}(\bar{Y}+\bar{\beta}))_{V}\right]_{x_{0}} .
$$

Indeed,

$$
\begin{aligned}
d \pi_{S_{0}}\left[(\mathbb{J}(X+\alpha))_{v},(\mathbb{J}(Y+\beta))_{v}\right]_{S_{0}}(f)= & d f_{\pi\left(S_{0}\right)}^{M} d \pi_{S_{0}}\left[(\mathbb{J}(X+\alpha))_{v},(\mathbb{J}(Y+\beta))_{v}\right]_{S_{0}} \\
= & d^{Z}(f \circ \pi)_{S_{0}}\left[(\mathbb{J}(X+\alpha))_{v},(\mathbb{J}(Y+\beta))_{v}\right]_{S_{0}} \\
= & \left((\mathbb{J}(X+\alpha))_{v}\right)_{S_{0}}\left((\mathbb{J}(Y+\beta))_{v}(f \circ \pi)\right) \\
& \left.-\left((\mathbb{J}(Y+\beta))_{v}\right)_{S_{0}}\left((\mathbb{J}(X+\alpha))_{v}\right)(f \circ \pi)\right) .
\end{aligned}
$$


Now, by (3),

$$
\begin{aligned}
& \left((\mathbb{J}(X+\alpha))_{v}\right)_{S_{0}}\left((\mathbb{J}(Y+\beta))_{v}(f \circ \pi)\right)=\left((\bar{S}(\bar{Y}+\bar{\beta}))_{v}\right)_{x_{0}}\left((\bar{S}(\bar{X}+\bar{\alpha}))_{v}(f)\right), \\
& \left((\mathbb{J}(Y+\beta))_{v}\right)_{S_{0}}\left((\mathbb{J}(X+\alpha))_{v}(f \circ \pi)\right)=\left((\bar{S}(\bar{X}+\bar{\alpha}))_{v}\right)_{x_{0}}\left((\bar{S}(\bar{Y}+\bar{\beta}))_{v}(f)\right) .
\end{aligned}
$$

Claim 2.

$$
\begin{aligned}
& \widetilde{d \pi}_{S_{0}} d^{Z}\left((\mathbb{J}(Y+\beta))_{f}\left((\mathbb{J}(X+\alpha))_{v}\right)\right)=d^{M}\left((\bar{S}(\bar{Y}+\bar{\beta}))_{f}\left((\bar{S}(\bar{X}+\bar{\alpha}))_{v}\right)\right)_{x_{0}}, \\
& \widetilde{d \pi}_{S_{0}} d^{Z}\left((\mathbb{J}(X+\alpha))_{f}\left((\mathbb{J}(Y+\beta))_{v}\right)\right)=d^{M}\left((\bar{S}(\bar{X}+\bar{\alpha}))_{f}\left((\bar{S}(\bar{Y}+\bar{\beta}))_{v}\right)\right)_{x_{0}} .
\end{aligned}
$$

Indeed,

$$
\begin{aligned}
& \left(\widetilde{d \pi}_{S_{0}}\left(d^{Z}\left((\mathbb{J}(Y+\beta))_{f}\left((\mathbb{J}(X+\alpha))_{V}\right)\right)\right)_{S_{0}}\right)\left(\bar{A}_{x_{0}}\right)=\left(d^{Z}\left((\mathbb{J}(Y+\beta))_{f}\left((\mathbb{J}(X+\alpha))_{V}\right)\right)\right)_{S_{0}}\left(A_{S_{0}}\right) \\
& =\lim _{t \rightarrow 0} \frac{1}{t}\left[(\mathbb{J}(Y+\beta))_{f}\left((\mathbb{J}(X+\alpha))_{v}\right)(\delta(t))-(\mathbb{J}(Y+\beta))_{f}\left((\mathbb{J}(X+\alpha))_{v}\right)(\delta(0))\right] \\
& =\lim _{t \rightarrow 0} \frac{1}{t}\left[\left(\widehat{d \pi}^{-1} \varphi(\delta(t)) \widehat{d \pi}\left(Y_{\delta(t)}+\beta_{\delta(t)}\right)\right)_{f}\left(\left(\widehat{d \pi}^{-1} \varphi(\delta(t)) \widehat{d \pi}\left(X_{\delta(t)}+\alpha_{\delta(t)}\right)\right)_{v}\right)\right. \\
& \left.-\left(\widehat{d \pi}^{-1} \varphi\left(S_{0}\right) \widehat{d \pi}\left(Y_{S_{0}}+\beta_{S_{0}}\right)\right)_{f}\left(\left(\widehat{d \pi}^{-1} \varphi\left(S_{0}\right) \widehat{d \pi}\left(X_{S_{0}}+\alpha_{S_{0}}\right)\right)_{v}\right)\right] \\
& =\lim _{t \rightarrow 0} \frac{1}{t}\left[\left(\widehat{d \pi}^{-1} \varphi(S(\bar{\delta}(t)))\left(\bar{Y}_{\bar{\delta}(t)}+\bar{\beta}_{\bar{\delta}(t)}\right)\right)_{f}\left(\left(\widehat{d \pi}^{-1} \varphi(S(\bar{\delta}(t)))\left(\bar{X}_{\bar{\delta}(t)}+\bar{\alpha}_{\bar{\delta}(t)}\right)\right)_{v}\right)\right. \\
& \left.-\left(\widehat{d \pi}^{-1} \varphi\left(S_{0}\right)\left(\bar{Y}_{x_{0}}+\bar{\beta}_{x_{0}}\right)\right)_{f}\left(\left(\widehat{d \pi}^{-1} \varphi\left(S_{0}\right)\left(\bar{X}_{x_{0}}+\bar{\alpha}_{x_{0}}\right)\right)_{v}\right)\right] \\
& =\lim _{t \rightarrow 0} \frac{1}{t}\left[\left(\varphi(S(\bar{\delta}(t)))\left(\bar{Y}_{\bar{\delta}(t)}+\bar{\beta}_{\bar{\delta}(t)}\right)\right)_{f}\left(\left(\varphi(S(\bar{\delta}(t)))\left(\bar{X}_{\bar{\delta}(t)}+\bar{\alpha}_{\bar{\delta}(t)}\right)\right)_{v}\right)\right. \\
& \left.-\left(\varphi\left(S_{0}\right)\left(\bar{Y}_{x_{0}}+\bar{\beta}_{x_{0}}\right)\right)_{f}\left(\left(\varphi\left(S_{0}\right)\left(\bar{X}_{x_{0}}+\bar{\alpha}_{x_{0}}\right)\right)_{v}\right)\right] \\
& =\lim _{t \rightarrow 0} \frac{1}{t}\left[\left(\bar{S}_{\bar{\delta}(t)}\left(\bar{Y}_{\bar{\delta}(t)}+\bar{\beta}_{\bar{\delta}(t)}\right)\right)_{f}\left(\left(\bar{S}_{\bar{\delta}(t)}\left(\bar{X}_{\bar{\delta}(t)}+\bar{\alpha}_{\bar{\delta}(t)}\right)\right)_{v}\right)\right. \\
& \left.-\left(\bar{S}_{x_{0}}\left(\bar{Y}_{x_{0}}+\bar{\beta}_{x_{0}}\right)\right)_{f}\left(\left(\bar{S}_{x_{0}}\left(\bar{X}_{x_{0}}+\bar{\alpha}_{x_{0}}\right)\right)_{v}\right)\right] \\
& =\lim _{t \rightarrow 0} \frac{1}{t}\left[(\varphi(\bar{S})(\bar{Y}+\bar{\beta}))_{f}\left((\varphi(\bar{S})(\bar{X}+\bar{\alpha}))_{v}\right)(\bar{\delta}(t))\right. \\
& \left.-(\bar{S}(\bar{Y}+\bar{\beta}))_{f}\left((\bar{S}(\bar{X}+\bar{\alpha}))_{v}\right)\left(x_{0}\right)\right] \\
& =d^{M}(\bar{S}(\bar{Y}+\bar{\beta}))_{f}\left((\bar{S}(\bar{X}+\bar{\alpha}))_{v}\right)\left(\bar{A}_{x_{0}}\right) \text {. }
\end{aligned}
$$

Claim 3.

$$
\begin{gathered}
\widetilde{d \pi}_{S_{0}}\left(\mathcal{L}_{(\mathbb{J}(X+\alpha))_{v}}(\mathbb{J}(Y+\beta))_{f}\right)_{S_{0}}=\left(\mathcal{L}_{(\bar{S}(\bar{X}+\bar{\alpha}))_{v}}(\bar{S}(\bar{Y}+\bar{\beta}))_{f}\right)_{x_{0}}, \\
\widetilde{d \pi}_{S_{0}}\left(L_{(\mathbb{J}(Y+\beta))_{v}}(\mathbb{J}(X+\alpha))_{f}\right)_{S_{0}}=\left(\mathcal{L}_{(\bar{S}(\bar{Y}+\bar{\beta}))_{v}}(\bar{S}(\bar{X}+\bar{\alpha}))_{f}\right)_{\chi_{0}} .
\end{gathered}
$$

Indeed,

$$
\begin{aligned}
\widetilde{d \pi}_{S_{0}}\left(\mathcal{L}_{(\mathbb{J}(X+\alpha))_{v}}(\mathbb{J}(Y+\beta))_{f}\right)_{S_{0}}\left(\bar{A}_{x_{0}}\right) & =\left(\mathcal{L}_{(\mathbb{J}(X+\alpha))_{v}}(\mathbb{J}(Y+\beta))_{f}\right)_{S_{0}}\left(A_{S_{0}}\right) \\
& =d^{Z}\left((\mathbb{J}(X+\alpha))_{v}(\mathbb{J}(Y+\beta))_{f}\right)_{S_{0}}\left(A_{S_{0}}\right)+\left(d^{Z}(\mathbb{J}(Y+\beta))_{f}\right)_{S_{0}}\left(\left((\mathbb{J}(X+\alpha))_{v}\right)_{S_{0}}, A_{S_{0}}\right) .
\end{aligned}
$$

We only have to examine the second term

$$
\begin{aligned}
\left(d^{Z}(\mathbb{J}(Y+\beta))_{f}\right)_{S_{0}}\left(\left((\mathbb{J}(X+\alpha))_{v}\right)_{S_{0}}, A_{S_{0}}\right)= & \left((\mathbb{J}(X+\alpha))_{v}\right)_{S_{0}}\left((\mathbb{J}(Y+\beta))_{f}(A)\right)-A_{S_{0}}\left((\mathbb{J}(Y+\beta))_{f}\left((\mathbb{J}(X+\alpha))_{v}\right)\right. \\
& -\left((\mathbb{J}(Y+\beta))_{f}\right)_{S_{0}}\left(\left[(\mathbb{J}(X+\alpha))_{v}, A\right]_{S_{0}}\right) .
\end{aligned}
$$

Let $\mu(t)$ be a horizontal curve contained in the image of $S(x)$ such that $\mu(0)=S_{0}$ and $\dot{\mu}(0)=\left((\mathbb{J}(X+\alpha))_{v}\right)_{S_{0}}$, and $\bar{\mu}(t)=\pi(\mu(t))$ the corresponding curve on $M$. Note that

$$
\begin{aligned}
\dot{\bar{\mu}}(0) & =d \pi_{S_{0}} \dot{\mu}(0) \\
& =d \pi_{S_{0}}\left(\left((\mathbb{J}(X+\alpha))_{v}\right)_{S_{0}}\right)
\end{aligned}
$$




$$
\begin{aligned}
& =\left(\widehat{d \pi}_{S_{0}} \widehat{d \pi}_{S_{0}}^{-1} \varphi\left(S_{0}\right) \widehat{d \pi}_{S_{0}}\left(X_{S_{0}}+\alpha_{S_{0}}\right)\right)_{v} \\
& =\left(\bar{S}_{X_{0}}\left(\bar{X}_{X_{0}}+\bar{\alpha}_{x_{0}}\right)\right)_{v} .
\end{aligned}
$$

Now,

$$
\begin{aligned}
\left((\mathbb{J}(X+\alpha))_{v}\right)_{S_{0}}\left((\mathbb{J}(Y+\beta))_{f}(A)\right) & =\lim _{t \rightarrow 0} \frac{1}{t}\left(\left((\mathbb{I}(Y+\beta))_{f}(A)\right)_{\mu(t)}-\left((\mathbb{J}(Y+\beta))_{f}(A)\right)_{S_{0}}\right) \\
& =\lim _{t \rightarrow 0} \frac{1}{t}\left(\left(\left(\widehat{d \pi}_{\mu(t)}^{-1} \varphi(\mu(t)) \widehat{d \pi}_{\mu(t)}\left(Y_{\mu(t)}+\beta_{\mu(t)}\right)\right)_{f}\left(A_{\mu(t)}\right)\right)-\left(\left(\widehat{d \pi}_{S_{0}}^{-1} \varphi\left(S_{0}\right) \widehat{d \pi}_{S_{0}}\left(Y_{S_{0}}+\beta_{S_{0}}\right)\right)_{f}\left(A_{S_{0}}\right)\right)\right) \\
& =\lim _{t \rightarrow 0} \frac{1}{t}\left(\left(\left(\varphi(S(\bar{\mu}(t)))\left(\bar{Y}_{\bar{\mu}(t)}+\bar{\beta}_{\bar{\mu}(t)}\right)\right)_{f}\left(\bar{A}_{\bar{\mu}(t)}\right)\right)-\left(\left(\varphi\left(S_{0}\right)\left(\bar{Y}_{x_{0}}+\bar{\beta}_{x_{0}}\right)\right)_{f}\left(\bar{A}_{x_{0}}\right)\right)\right) \\
& =\lim _{t \rightarrow 0} \frac{1}{t}\left(\left((\bar{S}(\bar{Y}+\bar{\beta}))_{f}(\bar{A})\right)(\bar{\mu}(t))-\left((\bar{S}(\bar{Y}+\bar{\beta}))_{f}(\bar{A})\right)\left(x_{0}\right)\right) \\
& =\left(\bar{S}_{x_{0}}\left(\bar{X}_{x_{0}}+\bar{\alpha}_{x_{0}}\right)\right)_{v}\left((\bar{S}(\bar{Y}+\bar{\beta}))_{f}(\bar{A})\right),
\end{aligned}
$$

and

$$
\begin{aligned}
\left((\mathbb{J}(Y+\beta))_{f}\right)_{S_{0}}\left(\left[(\mathbb{J}(X+\alpha))_{v}, A\right]_{S_{0}}\right) & =\left({\widehat{d \pi_{S}}}_{S_{0}}^{-1} \varphi\left(S_{0}\right) \widehat{d \pi}_{S_{0}}\left(Y_{S_{0}}+\beta_{S_{0}}\right)\right)_{f}\left(\left[(\mathbb{J}(X+\alpha))_{v}, A\right]_{S_{0}}\right) \\
& =\left(\bar{S}_{x_{0}}\left(\bar{Y}_{x_{0}}+\bar{\beta}_{x_{0}}\right)\right)_{f}\left(d \pi_{S_{0}}\left(\left[(\mathbb{J}(X+\alpha))_{v}, A\right]_{S_{0}}\right)\right) \\
& \left.\left.=\left(\bar{S}_{x_{0}}\left(\bar{Y}_{x_{0}}+\bar{\beta}_{x_{0}}\right)\right)_{f}\left(\left[(\bar{S}(\bar{X}+\bar{\alpha}))_{v}\right), \bar{A}\right)\right]_{x_{0}}\right),
\end{aligned}
$$

which proves Claim 3, Lemma 5.9 and 5.6.

If $\mathcal{T}$ satisfies condition (2), by Lemma 2.1

$$
\begin{aligned}
\widehat{d \pi}_{S_{0}} \mathcal{N}^{\mathbb{J}}(X+\alpha, Y+\beta)_{S_{0}} & =\left(\mathcal{N}^{\bar{S}}(\bar{X}+\bar{\alpha}, \bar{Y}+\bar{\beta})\right)_{x_{0}} \\
& =0,
\end{aligned}
$$

which concludes the proof of Lemma 5.4.

\subsubsection{Proof of Lemma 5.5}

Recall that

$$
\mathcal{N}^{\rrbracket}(X+\alpha, Y+\beta)^{v}=\llbracket \mathbb{J}(X+\alpha), \mathbb{J}(Y+\beta) \rrbracket^{v}-\mathbb{J} \llbracket \rrbracket(X+\alpha), Y+\beta \rrbracket^{v}-\mathbb{J} \llbracket X+\alpha, \mathbb{J}(Y+\beta) \rrbracket^{v}-\llbracket X+\alpha, Y+\beta \rrbracket^{v} .
$$

As before, we will compute one term at a time. In the following calculations we will continue to consider $X+\alpha$ and $Y+\beta$ to be basic fields, and $U$ be a local vertical vector field around $S_{0} \in Z$.

\section{Lemma 5.10.}

$$
\begin{aligned}
\llbracket \mathbb{J}(X+\alpha), \mathbb{J}(Y+\beta) \rrbracket_{S_{0}}^{v}= & -R\left((\bar{S}(\bar{X}+\bar{\alpha}))_{v},(\bar{S}(\bar{Y}+\bar{\beta}))_{v}\right)_{S_{0}}-\left\langle\bar{Y}+\bar{\beta}, \varphi\left(S_{0}\right) \varphi(\cdot)(\bar{X}+\bar{\alpha})\right\rangle_{x_{0}} \\
& +\left\langle\varphi\left(S_{0}\right) \varphi(\cdot)(\bar{Y}+\bar{\beta}), \bar{X}+\bar{\alpha}\right\rangle_{x_{0}},
\end{aligned}
$$

Proof. We have

$$
\begin{aligned}
\llbracket \mathbb{J}(X+\alpha), \mathbb{J}(Y+\beta) \rrbracket_{S_{0}}^{v}= & {\left[(\mathbb{J}(X+\alpha))_{v},(\mathbb{J}(Y+\beta))_{v}\right]_{S_{0}}^{v}+\left(\mathcal{L}_{(\mathbb{J}(X+\alpha))_{v}}(\mathbb{J}(Y+\beta))_{f}\right)_{S_{0}}^{v}-\left(\mathcal{L}_{(\mathbb{J}(Y+\beta))_{v}}(\mathbb{J}(X+\alpha))_{f}\right)_{S_{0}}^{v} } \\
& -\frac{1}{2}\left(d\left((\mathbb{J}(Y+\beta))_{f}\left((\mathbb{J}(X+\alpha))_{v}\right)\right)_{S_{0}}^{v}+\frac{1}{2}\left(d\left((\mathbb{J}(X+\alpha))_{f}\left((\mathbb{J}(Y+\beta))_{v}\right)\right)_{S_{0}}^{v}\right.\right.
\end{aligned}
$$

Claim 1. By Lemma 4.5,

$$
\left[(\mathbb{J}(X+\alpha))_{v},(\mathbb{J}(Y+\beta))_{v}\right]_{S_{0}}^{v}=-R\left(\left(\varphi\left(S_{0}\right)(\bar{X}+\bar{\alpha})\right)_{v},\left(\varphi\left(S_{0}\right)(\bar{Y}+\bar{\beta})\right)_{v}\right) S_{0}
$$


Claim 2.

$$
\begin{aligned}
& \left(\mathcal{L}_{(\mathbb{J}(X+\alpha))_{v}}(\mathbb{J}(Y+\beta))_{f}\right)_{S_{0}}^{v}\left(U_{S_{0}}\right)=\left(\varphi\left(S_{0}\right)\left(\bar{Y}_{X_{0}}+\bar{\beta}_{x_{0}}\right)\right)_{f}\left(\left(\varphi\left(U_{S_{0}}\right)\left(\bar{X}_{X_{0}}+\bar{\alpha}_{x_{0}}\right)\right)_{v}\right), \\
& \left(\mathcal{L}_{(\mathbb{J}(Y+\beta))_{v}}(\mathbb{J}(X+\alpha))_{f}\right)_{S_{0}}^{v}\left(U_{S_{0}}\right)=\left(\varphi\left(S_{0}\right)\left(\bar{X}_{X_{0}}+\bar{\alpha}_{x_{0}}\right)\right)_{f}\left(\left(\varphi\left(U_{S_{0}}\right)\left(\bar{Y}_{X_{0}}+\bar{\beta}_{X_{0}}\right)\right)_{v}\right) .
\end{aligned}
$$

Indeed, by the proof of Claim 4 of Lemma 5.2

$$
\begin{aligned}
\left(\mathcal{L}_{(\mathbb{J}(X+\alpha))_{v}}(\mathbb{J}(Y+\beta))_{f}\right)_{S_{0}}^{v}\left(U_{S_{0}}\right)= & (\mathbb{J}(X+\alpha))_{v}\left((\mathbb{J}(Y+\beta))_{f}(U)\right)_{S_{0}} \\
& -(\mathbb{J}(Y+\beta))_{f}\left(\left[(\mathbb{J}(X+\alpha))_{v}, U\right]_{S_{0}}\right) \\
= & -(\mathbb{J}(Y+\beta))_{f}\left(\left[(\mathbb{J}(X+\alpha))_{v}, U\right]_{S_{0}}\right) \\
= & (\mathbb{J}(Y+\beta))_{f}\left(\left[U,(\mathbb{J}(X+\alpha))_{v}\right]_{S_{0}}\right) \\
= & \left((\mathbb{J}(Y+\beta))_{f}\right)_{S_{0}}\left(\mathcal{L}_{U}(\mathbb{J}(X+\alpha))_{v}\right)_{S_{0}} \\
= & \left(\varphi\left(S_{0}\right)\left(\bar{Y}_{X_{0}}+\bar{\beta}_{X_{0}}\right)\right)_{f}\left(\left(\varphi\left(U_{S_{0}}\right)\left(\bar{X}_{X_{0}}+\bar{\alpha}_{X_{0}}\right)\right)_{v}\right) .
\end{aligned}
$$

\section{Claim 3.}

$$
\begin{aligned}
d\left((\mathbb{J}(Y+\beta))_{f}(\mathbb{J}(X+\alpha))_{v}\right)_{S_{0}}^{v}\left(U_{S_{0}}\right)= & \left(\left(\varphi\left(S_{0}\right)\left(\bar{Y}_{x_{0}}+\bar{\beta}_{x_{0}}\right)\right)_{f}\left(\left(\varphi\left(U_{S_{0}}\right)\left(\bar{X}_{x_{0}}+\bar{\alpha}_{x_{0}}\right)\right)_{v}\right)\right) \\
& \left.+\left(\left(\varphi\left(U_{S_{0}}\right)\left(\bar{X}_{x_{0}}+\bar{\alpha}_{x_{0}}\right)\right)_{f}\right)\left(\varphi\left(S_{0}\right)\left(\bar{Y}_{x_{0}}+\bar{\beta}_{x_{0}}\right)\right)_{v}\right), \\
d\left((\mathbb{J}(X+\alpha))_{f}(\mathbb{J}(Y+\beta))_{v}\right)_{S_{0}}^{v}\left(U_{S_{0}}\right)= & \left(\left(\varphi\left(S_{0}\right)\left(\bar{X}_{x_{0}}+\bar{\alpha}_{x_{0}}\right)\right)_{f}\left(\left(\varphi\left(U_{S_{0}}\right)\left(\bar{Y}_{x_{0}}+\bar{\beta}_{x_{0}}\right)\right)_{v}\right)\right) \\
+ & \left.\left(\left(\varphi\left(U_{S_{0}}\right)\left(\bar{X}_{x_{0}}+\bar{\alpha}_{x_{0}}\right)\right)_{f}\right)\left(\varphi\left(S_{0}\right)\left(\bar{Y}_{x_{0}}+\bar{\beta}_{x_{0}}\right)\right)_{v}\right) .
\end{aligned}
$$

Indeed

$$
\begin{aligned}
& d\left((\mathbb{J}(Y+\beta))_{f}(\mathbb{J}(X+\alpha))_{v}\right)_{S_{0}}^{v}\left(U_{S_{0}}\right) \\
& =d\left((\mathbb{J}(Y+\beta))_{f}(\mathbb{J}(X+\alpha))_{v}\right)_{S_{0}}\left(U_{S_{0}}\right) \\
& =\lim _{t \rightarrow 0} \frac{1}{t}\left((\mathbb{J}(Y+\beta))_{f}(\mathbb{J}(X+\alpha))_{v}\left(\phi_{t}^{U}\left(S_{0}\right)\right)-(\mathbb{J}(Y+\beta))_{f}(\mathbb{J}(X+\alpha))_{v}\left(S_{0}\right)\right) \\
& =\lim _{t \rightarrow 0} \frac{1}{t}\left(\left(\widehat{d \pi}_{\phi_{t}^{U}\left(S_{0}\right)}^{-1} \varphi\left(\phi_{t}^{U}\left(S_{0}\right)\right) \widehat{d \pi}_{\phi_{t}^{U}\left(S_{0}\right)}\left(Y_{\phi_{t}^{U}\left(S_{0}\right)}+\beta_{\phi_{t}^{U}\left(S_{0}\right)}\right)\right)_{f}\left(\widehat{d \pi}_{\phi_{t}^{U}\left(S_{0}\right)}^{-1} \varphi\left(\phi_{t}^{U}\left(S_{0}\right)\right) \widehat{d \pi}_{\phi_{t}^{U}\left(S_{0}\right)}\left(X_{\phi_{t}^{U}\left(S_{0}\right)}+\alpha_{\phi_{t}^{U}\left(S_{0}\right)}\right)\right)_{v}\right. \\
& \left.-\left(\widehat{d \pi}_{S_{0}}^{-1} \varphi\left(S_{0}\right) \widehat{d \pi}_{S_{0}}\left(Y_{S_{0}}+\beta_{S_{0}}\right)\right)_{f}\left(\widehat{d \pi}_{S_{0}}^{-1} \varphi\left(S_{0}\right) \widehat{d \pi}_{S_{0}}\left(X_{S_{0}}+\alpha_{S_{0}}\right)\right)_{v}\right) \\
& =\lim _{t \rightarrow 0} \frac{1}{t}\left(\left(\varphi\left(\phi_{t}^{U}\left(S_{0}\right)\right)\left(\bar{Y}_{x_{0}}+\bar{\beta}_{x_{0}}\right)\right)_{f}\left(\varphi\left(\phi_{t}^{U}\left(S_{0}\right)\right)\left(\bar{X}_{x_{0}}+\bar{\alpha}_{x_{0}}\right)\right)_{v}-\left(\varphi\left(S_{0}\right)\left(\bar{Y}_{x_{0}}+\bar{\beta}_{x_{0}}\right)\right)_{f}\left(\varphi\left(S_{0}\right)\left(\bar{X}_{x_{0}}+\bar{\alpha}_{x_{0}}\right)\right)_{v}\right) \\
& =\lim _{t \rightarrow 0} \frac{1}{t}\left(\left(\varphi\left(\phi_{t}^{U}\left(S_{0}\right)\right)\left(\bar{Y}_{x_{0}}+\bar{\beta}_{x_{0}}\right)\right)_{f}\left(\varphi\left(\phi_{t}^{U}\left(S_{0}\right)\right)\left(\bar{X}_{x_{0}}+\bar{\alpha}_{x_{0}}\right)\right)_{v}-\left(\varphi\left(\phi_{t}^{U}\left(S_{0}\right)\right)\left(\bar{Y}_{x_{0}}+\bar{\beta}_{x_{0}}\right)\right)_{f}\left(\varphi\left(S_{0}\right)\left(\bar{X}_{x_{0}}+\bar{\alpha}_{x_{0}}\right)\right)_{v}\right. \\
& \left.+\left(\varphi\left(\phi_{t}^{U}\left(S_{0}\right)\right)\left(\bar{Y}_{x_{0}}+\bar{\beta}_{x_{0}}\right)\right)_{f}\left(\varphi\left(S_{0}\right)\left(\bar{X}_{x_{0}}+\bar{\alpha}_{x_{0}}\right)\right)_{v}-\left(\varphi\left(S_{0}\right)\left(\bar{Y}_{x_{0}}+\bar{\beta}_{x_{0}}\right)\right)_{f}\left(\varphi\left(S_{0}\right)\left(\bar{X}_{x_{0}}+\bar{\alpha}_{x_{0}}\right)\right)_{v}\right) \\
& =\lim _{t \rightarrow 0} \frac{1}{t}\left(\left(\varphi\left(\phi_{t}^{U}\left(S_{0}\right)\right)\left(\bar{Y}_{x_{0}}+\bar{\beta}_{x_{0}}\right)\right)_{f}\left(\left(\left(\varphi\left(\phi_{t}^{U}\left(S_{0}\right)\right)-\varphi\left(S_{0}\right)\right)\left(\bar{X}_{x_{0}}+\bar{\alpha}_{x_{0}}\right)\right)_{v}\right)\right. \\
& \left.+\left(\left(\varphi\left(\phi_{t}^{U}\left(S_{0}\right)\right)\left(\bar{Y}_{x_{0}}+\bar{\beta}_{x_{0}}\right)\right)_{f}-\left(\varphi\left(S_{0}\right)\left(\bar{Y}_{x_{0}}+\bar{\beta}_{x_{0}}\right)\right)_{f}\right)\left(\varphi\left(S_{0}\right)\left(\bar{X}_{x_{0}}+\bar{\alpha}_{x_{0}}\right)\right)_{v}\right) \\
& =\left(( \varphi ( \operatorname { l i m } _ { t \rightarrow 0 } \frac { 1 } { t } \phi _ { t } ^ { U } ( S _ { 0 } ) ) ( \overline { Y } _ { x _ { 0 } } + \overline { \beta } _ { x _ { 0 } } ) ) _ { f } \left(\left(\left(\varphi\left(\lim _{t \rightarrow 0} \frac{1}{t}\left(\phi_{t}^{U}\left(S_{0}\right)-S_{0}\right)\right)\left(\bar{X}_{x_{0}}+\bar{\alpha}_{x_{0}}\right)\right)_{v}\right)\right.\right. \\
& +\left(\left(\left(\varphi\left(\lim _{t \rightarrow 0} \frac{1}{t}\left(\phi_{t}^{U}\left(S_{0}\right)-S_{0}\right)\right)\left(\bar{Y}_{x_{0}}+\bar{\beta}_{x_{0}}\right)\right)_{f}\right)\left(\varphi\left(S_{0}\right)\left(\bar{X}_{x_{0}}+\bar{\alpha}_{x_{0}}\right)\right)_{v}\right) \\
& \left.=\left(\left(\varphi\left(S_{0}\right)\left(\bar{Y}_{x_{0}}+\bar{\beta}_{x_{0}}\right)\right)_{f}\left(\left(\varphi\left(U_{S_{0}}\right)\left(\bar{X}_{x_{0}}+\bar{\alpha}_{x_{0}}\right)\right)_{v}\right)\right)+\left(\left(\varphi\left(U_{S_{0}}\right)\left(\bar{Y}_{x_{0}}+\bar{\beta}_{x_{0}}\right)\right)_{f}\right)\left(\varphi\left(S_{0}\right)\left(\bar{X}_{x_{0}}+\bar{\alpha}_{x_{0}}\right)\right)_{v}\right) \text {. }
\end{aligned}
$$

Adding up the results we get:

$$
\begin{aligned}
\llbracket \mathbb{J}(X+\alpha), \mathbb{J}(Y+\beta) \rrbracket_{S_{0}}^{v=} & R\left(\left(\varphi\left(S_{0}\right)\left(\bar{X}_{x_{0}}+\bar{\alpha}_{x_{0}}\right)\right)_{v},\left(\varphi\left(S_{0}\right)\left(\bar{Y}_{x_{0}}+\bar{\beta}_{x_{0}}\right)\right)_{v}\right)\left(S_{0}\right)+\left\langle\varphi\left(S_{0}\right)\left(\bar{Y}_{x_{0}}+\bar{\beta}_{x_{0}}\right), \varphi(\cdot)\left(\bar{X}_{x_{0}}+\bar{\alpha}_{x_{0}}\right)\right\rangle \\
& -\left\langle\varphi(\cdot)\left(\bar{Y}_{x_{0}}+\bar{\beta}_{x_{0}}\right), \varphi\left(S_{0}\right)\left(\bar{X}_{x_{0}}+\bar{\alpha}_{x_{0}}\right)\right\rangle
\end{aligned}
$$




$$
\begin{aligned}
= & R\left(\left(\varphi\left(S_{0}\right)\left(\bar{X}_{x_{0}}+\bar{\alpha}_{x_{0}}\right)\right)_{v},\left(\varphi\left(S_{0}\right)\left(\bar{Y}_{x_{0}}+\bar{\beta}_{x_{0}}\right)\right)_{v}\right)\left(S_{0}\right)-\left\langle\bar{Y}_{x_{0}}+\bar{\beta}_{x_{0}}, \varphi\left(S_{0}\right) \varphi(\cdot)\left(\bar{X}_{x_{0}}+\bar{\alpha}_{x_{0}}\right)\right\rangle \\
& +\left\langle\varphi\left(S_{0}\right) \varphi(\cdot)\left(\bar{Y}_{x_{0}}+\bar{\beta}_{x_{0}}\right), \bar{X}_{x_{0}}+\bar{\alpha}_{x_{0}}\right\rangle .
\end{aligned}
$$

since $\varphi\left(S_{0}\right)$ is an orthogonal generalized almost complex structure on $\mathbb{T} M$, which concludes the proof of Lemma 5.10.

\section{Lemma 5.11.}

$$
\begin{aligned}
& (-\mathbb{J} \llbracket \mathbb{\rrbracket}(X+\alpha), Y+\beta \rrbracket)_{S_{0}}^{v}=S_{0} \cdot R\left(\left(\varphi\left(S_{0}\right)\left(\bar{X}_{x_{0}}+\bar{\alpha}_{x_{0}}\right)\right)_{v}, \bar{Y}\right)\left(S_{0}\right)+\left\langle\bar{Y}_{x_{0}}+\bar{\beta}_{x_{0}}, \varphi\left(S_{0}\right) \varphi(\cdot)\left(\bar{X}_{x_{0}}+\bar{\alpha}_{x_{0}}\right)\right\rangle, \\
& (-\mathbb{J} \llbracket \mathbb{J}(Y+\beta), X+\alpha \rrbracket)_{S_{0}}^{v}=S_{0} \cdot R\left(\bar{X}_{x_{0}},\left(\varphi\left(S_{0}\right)\left(\bar{Y}_{x_{0}}+\bar{\beta}_{x_{0}}\right)\right)_{v}\right)\left(S_{0}\right)+\left\langle\bar{X}_{x_{0}}+\bar{\alpha}_{x_{0}}, \varphi\left(S_{0}\right) \varphi(\cdot)\left(\bar{Y}_{x_{0}}+\bar{\beta}_{x_{0}}\right)\right\rangle \text {. }
\end{aligned}
$$

Proof. We have

$$
\begin{aligned}
(-\mathbb{J} \llbracket \mathbb{J}(X+\alpha), Y+\beta \rrbracket)_{S_{0}}^{v}= & -I_{S_{0}}\left(\left[(\mathbb{J}(X+\alpha))_{v}, Y\right]_{S_{0}}^{v}\right)+I_{S_{0}}^{\star}\left(\mathcal{L}_{(\mathbb{J}(X+\alpha))_{v}} \beta\right)_{S_{0}}^{v}-I_{S_{0}}^{\star}\left(\mathcal{L}_{Y}(\mathbb{J}(X+\alpha))_{f}\right)_{S_{0}}^{v} \\
& -\frac{1}{2} I_{S_{0}}^{\star}\left(d\left(\beta\left((\mathbb{J}(X+\alpha))_{v}\right)\right)\right)_{S_{0}}^{v}+\frac{1}{2} I_{S_{0}}^{\star}\left(d\left((\mathbb{J}(X+\alpha))_{f}\right)(Y)\right)_{S_{0}}^{v} .
\end{aligned}
$$

Claim 1. By Lemma 4.5

$$
-I_{S_{0}}\left(\left[(J(X+\alpha))_{v}, Y\right]\right)_{S_{0}}^{v}=S_{0} \cdot R\left((\bar{S}(\bar{X}+\bar{\alpha}))_{v}, \bar{Y}\right) S_{0} .
$$

Claim 2.

$$
I_{S_{0}}\left(\mathcal{L}_{(\mathbb{J}(X+\alpha))_{v}} \beta\right)_{S_{0}}^{v}=\bar{\beta}_{x_{0}}\left(\left(\varphi\left(S_{0}\right) \varphi(\cdot)(\bar{X}+\bar{\alpha})_{\chi_{0}}\right)_{v}\right) .
$$

Indeed, by the proof of Claim 4 of Lemma 5.2

$$
\begin{aligned}
I_{S_{0}}\left(\mathcal{L}_{(\mathbb{J}(X+\alpha))_{v}} \beta\right)_{S_{0}}^{v}\left(U_{S_{0}}\right) & =\left((\mathbb{J}(X+\alpha))_{v}\right)_{S_{0}}(\beta(I U))_{S_{0}}-\beta_{S_{0}}\left[(\mathbb{J}(X+\alpha))_{v}, I U\right]_{S_{0}} \\
& =\beta_{S_{0}}\left[I U,(\mathbb{J}(X+\alpha))_{v}\right]_{S_{0}} \\
& =\beta_{S_{0}}\left(\mathcal{L}_{I U}(\mathbb{J}(X+\alpha))_{v}\right)_{S_{0}} \\
& =\bar{\beta}_{x_{0}}\left(\left(\varphi\left(S_{0}\right) \varphi\left(U_{S_{0}}\right)(\bar{X}+\bar{\alpha})_{x_{0}}\right)_{v}\right) .
\end{aligned}
$$

Claim 3. By Lemma 4.1,

$$
\left(-L_{Y}(\mathbb{J}(X+\alpha))_{f}\right)_{S_{0}}^{v}=0 .
$$

Claim 4.

$$
I_{S_{0}}^{\star}\left(d\left(\beta\left((\mathbb{J}(X+\alpha))_{v}\right)\right)\right)_{S_{0}}^{v}=\bar{\beta}_{x_{0}}\left(\left(\varphi\left(S_{0}\right) \varphi(\cdot)\left(\bar{X}_{x_{0}}+\bar{\alpha}_{x_{0}}\right)\right)_{V}\right) .
$$

Indeed,

$$
\begin{aligned}
I_{S_{0}}^{\star}\left(d\left(\beta\left((\mathbb{d}(X+\alpha))_{v}\right)\right)\right)_{S_{0}}^{v}\left(U_{S_{0}}\right)= & \left(d\left(\beta\left((\mathbb{J}(X+\alpha))_{v}\right)\right)\right)_{S_{0}}^{v}\left(I_{S_{0}} U_{S_{0}}\right) \\
= & \left(d\left(\beta\left((\mathbb{J}(X+\alpha))_{v}\right)\right)\right)_{S_{0}}\left(I_{S_{0}} U_{S_{0}}\right) \\
= & \lim _{t \rightarrow 0} \frac{1}{t}\left(\left(\beta\left((\mathbb{J}(X+\alpha))_{v}\right)\right)_{\phi_{t}^{I U}\left(S_{0}\right)}-\left(\beta\left((\mathbb{J}(X+\alpha))_{v}\right)\right)_{S_{0}}\right) \\
= & \lim _{t \rightarrow 0} \frac{1}{t}\left(\left(\bar{\beta}_{x_{0}} d \pi_{\phi_{t}^{I U}\left(S_{0}\right)}\left(\left(\widehat{d \pi}_{\phi_{t}^{I U}\left(S_{0}\right)}^{-1} \varphi\left(\phi_{t}^{I U}\left(S_{0}\right)\right) \widehat{d \pi}_{\phi_{t}^{I U}\left(S_{0}\right)}(X+\alpha)\right)_{v}\right)_{\phi_{t}^{I U}\left(S_{0}\right)}\right)\right. \\
& \left.-\left(\bar{\beta}_{x_{0}} d \pi_{S_{0}}\left(\left(\widehat{d \pi}_{S_{0}}^{-1} \varphi\left(S_{0}\right) \widehat{d \pi}_{S_{0}}(X+\alpha)\right)_{v}\right)_{S_{0}}\right)\right) \\
= & \lim _{t \rightarrow 0} \frac{1}{t}\left(\left(\bar{\beta}_{\chi_{0}}\left(\left(\varphi\left(\phi_{t}^{I U}\left(S_{0}\right)\right)\left(\bar{X}_{x_{0}}+\bar{\alpha}_{x_{0}}\right)\right)_{v}\right)\right)\right.
\end{aligned}
$$




$$
\begin{aligned}
& \left.-\left(\bar{\beta}_{x_{0}}\left(\left(\varphi\left(S_{0}\right)\left(\bar{X}_{x_{0}}+\bar{\alpha}_{x_{0}}\right)\right)_{v}\right)\right)\right) \\
= & \bar{\beta}_{x_{0}}\left(\left(\varphi\left(\lim _{t \rightarrow 0} \frac{1}{t}\left(\phi_{t}^{I U}\left(S_{0}\right)-S_{0}\right)\right)\left(\bar{X}_{x_{0}}+\bar{\alpha}_{x_{0}}\right)\right)_{v}\right) \\
= & \bar{\beta}_{\chi_{0}}\left(\left(\varphi\left(I_{S_{0}} U_{S_{0}}\right)\left(\bar{X}_{x_{0}}+\bar{\alpha}_{x_{0}}\right)\right)_{v}\right) \\
= & \bar{\beta}_{x_{0}}\left(\left(\varphi\left(S_{0}\right) \varphi\left(U_{S_{0}}\right)\left(\bar{X}_{x_{0}}+\bar{\alpha}_{x_{0}}\right)\right)_{v}\right) .
\end{aligned}
$$

Claim 5.

$$
I_{S_{0}}^{\star}\left(d\left((\mathbb{J}(X+\alpha))_{f}(Y)\right)_{S_{0}}\right)^{v}=\left(\varphi\left(S_{0}\right) \varphi(\cdot)\left(\bar{X}_{x_{0}}+\bar{\alpha}_{x_{0}}\right)\right)_{f}\left(\bar{Y}_{x_{0}}\right) .
$$

Indeed, by the proof of Claim 5 of Lemma 5.2

$$
\begin{aligned}
I_{S_{0}}^{\star}\left(d\left((\mathbb{J}(X+\alpha))_{f}(Y)\right)_{S_{0}}\right)^{v}\left(U_{S_{0}}\right) & =\left(d\left((\mathbb{J}(X+\alpha))_{f}(Y)\right)_{S_{0}}\right)^{v}\left(I_{S_{0}} U_{S_{0}}\right) \\
& =\left(d\left((\mathbb{J}(X+\alpha))_{f}(Y)\right)_{S_{0}}\right)\left(I_{S_{0}} U_{S_{0}}\right) \\
& =\left(\varphi\left(S_{0}\right) \varphi\left(U_{S_{0}}\right)(\bar{X}+\bar{\alpha})_{x_{0}}\right)_{f}\left(\bar{Y}_{X_{0}}\right) .
\end{aligned}
$$

which proves Claim 5 and Lemma 5.11.

\section{Lemma 5.12.}

$$
-\llbracket X+\alpha, Y+\beta \rrbracket_{S_{0}}^{v}=R(\bar{X}, \bar{Y})_{S_{0}}
$$

Proof. We have

$$
-\llbracket X+\alpha, Y+\beta \rrbracket_{S_{0}}^{v}=-[X, Y]_{S_{0}}^{v}\left(-\mathcal{L}_{X} \beta\right)_{S_{0}}^{v}+\left(\mathcal{L}_{Y} \alpha\right)_{S_{0}}^{v}+\frac{1}{2}(d \beta(X))_{S_{0}}^{v}-\frac{1}{2}(d \alpha(Y))_{S_{0}}^{v} .
$$

Claim 1. By Lemma 4.5,

$$
-[X, Y]_{S_{0}}^{V}=R(\bar{X}, \bar{Y})\left(S_{0}\right)
$$

Claim 2. By the Lemma 4.1,

$$
\begin{aligned}
& \left(\mathcal{L}_{X} \beta\right)_{S_{0}}^{v}=0, \\
& \left(\mathcal{L}_{Y} \alpha\right)_{S_{0}}^{v}=0 .
\end{aligned}
$$

Claim 3.

$$
\begin{aligned}
& (d(\beta(X)))_{S_{0}}^{v}=0, \\
& (d(\alpha(Y)))_{S_{0}}^{v}=0 .
\end{aligned}
$$

Indeed,

$$
\begin{aligned}
(d(\beta(X)))_{S_{0}}^{v}(U) & =(d(\beta(X)))_{S_{0}}(U) \\
& =\lim _{t \rightarrow 0} \frac{1}{t}\left(\beta(X)\left(\phi_{t}^{U}\left(S_{0}\right)\right)-\beta(X)\left(S_{0}\right)\right) \\
& =\lim _{t \rightarrow 0} \frac{1}{t}\left(\bar{\beta}_{x_{0}}\left(d \pi_{\phi_{t}^{U}\left(S_{0}\right)}\left(X_{\phi_{t}^{U}\left(S_{0}\right)}\right)\right)-\bar{\beta}_{x_{0}}\left(d \pi_{S_{0}}\left(X_{S_{0}}\right)\right)\right) \\
& =\lim _{t \rightarrow 0} \frac{1}{t}\left(\bar{\beta}_{x_{0}}\left(\bar{X}_{x_{0}}\right)-\bar{\beta}_{x_{0}}\left(\bar{X}_{x_{0}}\right)\right) \\
& =0 .
\end{aligned}
$$


Now,

$$
\begin{aligned}
& \llbracket \mathbb{J}(X+\alpha), \mathbb{J}(Y+\beta) \rrbracket_{S_{0}}^{v}+(-\mathbb{J} \llbracket \mathbb{J}(X+\alpha), Y+\beta \rrbracket)_{S_{0}}^{v}+(-\mathbb{J} \llbracket X+\alpha, \mathbb{J}(Y+\beta) \rrbracket)_{S_{0}}^{v}+(-\llbracket X+\alpha, Y+\beta \rrbracket)_{S_{0}}^{v} \\
= & -R\left(\left(\varphi\left(S_{0}\right)\left(\bar{X}_{x_{0}}+\bar{\alpha}_{x_{0}}\right)\right)_{v},\left(\varphi\left(S_{0}\right)\left(\bar{Y}_{x_{0}}+\bar{\beta}_{x_{0}}\right)\right)_{v}\right)\left(S_{0}\right)-\left\langle\bar{Y}_{x_{0}}+\bar{\beta}_{x_{0}}, \varphi\left(S_{0}\right) \varphi(\cdot)\left(\bar{X}_{x_{0}}+\bar{\alpha}_{x_{0}}\right)\right\rangle \\
& +\left\langle\varphi\left(S_{0}\right) \varphi(\cdot)\left(\bar{Y}_{x_{0}}+\bar{\beta}_{x_{0}}\right), \bar{X}_{x_{0}}+\bar{\alpha}_{x_{0}}\right\rangle+S_{0} \cdot R\left(\left(\varphi\left(S_{0}\right)\left(\bar{X}_{x_{0}}+\bar{\alpha}_{x_{0}}\right)\right)_{v}, \bar{Y}_{x_{0}}\right)\left(S_{0}\right) \\
& +\left\langle\bar{Y}_{x_{0}}+\bar{\beta}_{x_{0}}, \varphi\left(S_{0}\right) \varphi(\cdot)\left(\bar{X}_{x_{0}}+\bar{\alpha}_{x_{0}}\right)\right\rangle+S_{0} \cdot R\left(\bar{X}_{x_{0}},\left(\varphi\left(S_{0}\right)\left(\bar{Y}_{x_{0}}+\bar{\beta}_{x_{0}}\right)\right)_{v}\right)\left(S_{0}\right) \\
& -\left\langle\varphi\left(S_{0}\right) \varphi(\cdot)\left(\bar{Y}_{x_{0}}+\bar{\beta}_{x_{0}}\right), \bar{X}_{x_{0}}+\bar{\alpha}_{x_{0}}\right\rangle+R\left(\bar{X}_{x_{0}}, \bar{Y}_{x_{0}}\right)\left(S_{0}\right) \\
= & -R\left(\left(\varphi\left(S_{0}\right)\left(\bar{X}_{x_{0}}+\bar{\alpha}_{x_{0}}\right)\right)_{v},\left(\varphi\left(S_{0}\right)\left(\bar{Y}_{x_{0}}+\bar{\beta}_{x_{0}}\right)\right)_{v}\right)\left(S_{0}\right)+S_{0} \cdot R\left(\left(\varphi\left(S_{0}\right)\left(\bar{X}_{x_{0}}+\bar{\alpha}_{x_{0}}\right)\right)_{v}, \bar{Y}_{x_{0}}\right)\left(S_{0}\right) \\
& +S_{0} \cdot R\left(\bar{X}_{x_{0}},\left(\varphi\left(S_{0}\right)\left(\bar{Y}_{x_{0}}+\bar{\beta}_{x_{0}}\right)\right)_{v}\right)\left(S_{0}\right)+R\left(\bar{X}_{x_{0}}, \bar{Y}_{x_{0}}\right)\left(S_{0}\right), \\
= & 0
\end{aligned}
$$

if the curvature satisfies conditon (1). This concludes the proof of Lemmas 5.5, 5.3 and Theorem 5.1.

We conclude by pointing out that it would be interesting to explore the construction of a Clifford diamond in the generalized setup analogous to that of the classical one [6]

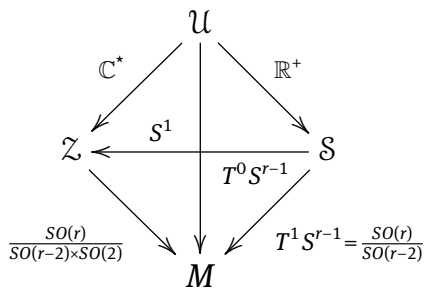

since the analogous fibrations do exist. One could study the naturally induced generalized structures and their relation to other generalized geometries, such as generalized contact structures, etc.

Acknowledgements: The first author wishes to thank the Centro de Investigación en Matemáticas for its hospitality and support. The authors also wish to thank the International Centre for Theoretical Physics for its hospitality.

Luis Fernando Hernández-Moguel and Rafael Herrera were partially supported by CONACYT grants CB256126 and A1-S-45886.

\section{References}

[1] Andriot, D.; Minasian, R.; Petrini, M.: Flux backgrounds from Twists. J. High Energy Phys. 2009, no. 12, 028, 37 pp.

[2] Arizmendi, G., Garcia-Pulido, A.L., Herrera R.: A note on the geometry and topology of almost even-Clifford Hermitian manifolds, Q. J. Math. 69 (2018), 321-376.

[3] Arizmendi G., Herrera R.: Centralizers of spin subalgebras, J. Geom. Phys. 97 (2015), 77-92.

[4] Arizmendi G., Herrera R., Santana N.: Almost even-Clifford hermitian manifolds with a large automorphism group, Proc. Amer. Math. Soc. 144 (2016), 4009-4020.

[5] Arizmendi, G., Hadfield, Ch.: Twistor spaces of Riemannian manifolds with even Clifford structures, Ann. Glob. Anal. Geom. (2017) 51, 11-20.

[6] Arizmendi, G.; Herrera, R.; Piccinni, P.: An even Clifford diamond. Ann Glob Anal Geom 57, 465-487 (2020).

[7] Bailey, M.; Gualtieri, M.: Local analytic geometry of generalized complex structures. Bull. London Math. Soc. 49: 307-319 (2017)

[8] Ben-Bassat, B.: Mirror symmetry and generalized complex manifolds. I. The transform on vector bundles, spinors, and branes, J. Geom. Phys. 56 (2006), no. 4, 533-558

[9] Besse, A.: Einstein manifolds, Ergebnisse der Mathematik, Vol. 10, Springer-Verlag, 1987.

[10] Bergery, L. B.: Variétés quaternionniennes. notes d' une conference á la table ronde “Variétés d' Einstein", Espalion (1979). (Unpublished).

[11] Barberis M.L., Dotti Miatello I.G., Miatello R.J.: On certain locally homogeneous Clifford manifolds, Ann. Global Anal. Geom. 13 (1995), 289-301.

[12] Burdujan, I.: On almost Cliffordian manifolds, Ital. J. Pure Appl. Math. (2003), 129-144. 
[13] Burdujan, I.: Clifford-Kähler manifolds, Balkan J. Geom. Appl. 13 (2008), 12-23.

[14] Cattaneo, A., Qiub, J., Zabzine, M.: 2D and 3D topological field theories for generalized complex geometry, Adv. Theor. Math. Phys. 14,695-725 (2010)

[15] Console, S., Olmos, C.: Clifford systems, algebraically constant second fundamental form and isoparametric hypersurfaces, Manuscripta Math. 97 (1998), 335-342.

[16] Davidov, J.: Generalized metrics and generalized twistor spaces Mathematische Zeitschrift 291, pages17-46 (2019)

[17] Deschamps, G.: Twistor space of a generalized quaternionic manifold. arXiv:1601.03882

[18] Ferus, D., Karcher, H., Münzner, H.F.: Cliffordalgebren und neue isoparametrische Hyperflächen, Math. Z. 177 (1981), 479502.

[19] Friedrich, T.: Dirac operators in Riemannian geometry. Translated from the 1997 German original by Andreas Nestke. Graduate Studies in Mathematics, 25. American Mathematical Society, Providence, RI, 2000. xvi+195 pp. ISBN: 0-8218-2055-9.

[20] Gualtieri, M.: Generalized complex geometry. PhD thesis, University of Oxford 2003.

[21] Gualtieri, M.: Branes on Poisson varieties. The many Facets of Geometry 1 (9), 368-395 (2010).

[22] Friedrich, Th.: Weak Spin(9)-structures on 16-dimensional Riemannian manifolds, Asian J. Math. 5 (2001), 129-160.

[23] Garcia-Pulido, A.L., Herrera, R.: Rigidity and vanishing theorems for almost even-Clifford Hermitian manifolds, SIGMA 13 (2017), 027, 28 pages.

[24] Graña, M.; Minasian, R.; Petrini, M.; Waldram, D.: T-duality, generalized geometry and non-geometric backgrounds, J. High Energy Phys. 2009, no. 4, 075, 39 pp.

[25] Hadfield C., Moroianu A.: Local geometry of even Clifford structures on conformal manifolds,Ann. Global Anal. Geom. 54 (2018), 301-313.

[26] Hitchin, N.: Lectures on generalized geometry. Surveys in Differential Geometry Volume 16 (2011) p. 79-124 https://dx.doi.org/10.4310/SDG.2011.v16.n1.a3

[27] Hull, C.: Generalised Geometry for M-Theory, J. High Energy Phys. 2007, no. 7, 079, 31 pp.

[28] Joyce, D.D.: Manifolds with many complex structures, Quart. J. Math. Oxford Ser. (2) 46 (1995), 169-184.

[29] Koepsell, K., Nicolai,H.; Samtleben, H.: An exceptional geometry for $d=11$ supergravity, Class. Quant. Grav. 17:3689-3702, 2000

[30] Koerber, P.: Lectures on Generalized Complex Geometry for Physicists, Fortsch. Phys. 59: 169-242, 2011

[31] Moroianu, A., Semmelmann, U.: Clifford structures on Riemannian manifolds. Adv. Math. 228 (2011), no. 2, 940-967.

[32] Moroianu A., Pilca, M.: Higher rank homogeneous Clifford structures, J. Lond. Math. Soc. 87 (2013), 384-400.

[33] Nikolayevsky, Y.: Osserman manifolds and Clifford structures, Houston J. Math. 29 (2003), 59-75.

[34] Pantilie, R.: Generalized quaternionic manifolds. Ann. Mat. Pura Appl. (4) 193, 633-641, (2014).

[35] Parton M., Piccinni P.: Spin(9) and almost complex structures on 16-dimensional manifolds, Ann. Global Anal. Geom. 41 (2012), 321-345.

[36] Parton M., Piccinni P.: The even Clifford structure of the fourth Severi variety, Complex Manifolds 2 (2015), 89-104.

[37] Parton M., Piccinni P., Vuletescu V.: Clifford systems in octonionic geometry, Rend. Semin. Mat. Univ. Politec. Torino 74 (2016). 269-290,

[38] Piccinni P.: On some Grassmannians carrying an even Clifford structure, Differential Geom. Appl. 59 (2018), 122-137.

[39] Pacheco, P. P., Waldram, D.: M-theory, exceptional generalised geometry and superpotentials, J. High Energy Phys. 2008, no. 9, 123, $37 \mathrm{pp}$

[40] Salamon, S.: Quaternionic Kähler manifolds. Invent. Math. 67, 143-171 (1982).

[41] Spindel P., Sevrin A., Troost W., Van Proeyen A.: Extended supersymmetric $\sigma$-models on group manifolds. I. The complex structures, Nuclear Phys. B 308 (1988), 662-698.

[42] Walschap, G.: Metric Structures in Differential Geometry. Graduate Studies in Mathematics, Springer-Verlag.

[43] Zambon, M.: Reduction of branes in generalized complex geometry. J.Symplectic Geom 6, no. 4 (2008), 353-378. 